\title{
THE POSITIVE REAL CONTROL PROBLEM AND THE GENERALIZED ALGEBRAIC RICCATI EQUATION FOR DESCRIPTOR SYSTEMS
}

\author{
He-Sheng Wang \\ Center for Aviation and Space Technology \\ Industrial Technology Research Institute \\ Hsinchu, Taiwan 310, R.O.C. \\ Chee-Fai Yung* \\ Department of Electrical Engineering \\ National Taiwan Ocean University \\ Keelung, Taiwan 202, R.O.C. \\ Fan-Ren Chang \\ Department of Electrical Engineering \\ National Taiwan University \\ Taipei, Taiwan 106, R.O.C.
}

\begin{abstract}
Key Words: positive real control, descriptor system, generalized algebraic Riccati equation.
\end{abstract}

\begin{abstract}
In this paper, some useful properties of generalized algebraic Riccati equations and generalized positive real lemma for descriptor systems are given. Based on these results, the main purpose of this paper is to investigate the positive real (PR) control problem for descriptor systems. Necessary and sufficient conditions are derived for the solution to this problem expressed in terms of two generalized algebraic Riccati equations which may be considered to be generalizations of the Riccati equations obtained by Sun et al. (1994). When these conditions hold, state space formulae for all controllers solving the problem are also given.
\end{abstract}

\section{INTRODUCTION}

Consider the standard block diagram shown in Fig. 1. In our problem, the plant $G$ is a descriptor system described by the following dynamical equations:

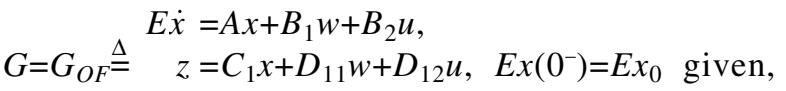

$$
\begin{aligned}
& y=C_{2} x+D_{21} w \text {, }
\end{aligned}
$$

(the subscript "OF" stands for output feedback) where $x \in \mathbb{R}^{n}$ is the state, and $w \in \mathbb{R}^{p}$ represents a set of exogenous inputs which includes disturbances to be rejected and/or reference commands to be tracked. $z \in \mathbb{R}^{p}$ is the output to be controlled and $y \in \mathbb{I}^{l}$ is the measured output. $u \in \mathbb{R}^{m}$ is the control input. $A, B_{1}$, $B_{2}, C_{1}, C_{2}, D_{11}, D_{12}$, and $D_{21}$ are constant matrices with compatible dimensions. $E \in \mathbb{R}^{n \times n}$ and $\operatorname{rank} E=r$ $<n$.

The controller $K$ is, in general, also a descriptor

*Correspondence addressee 


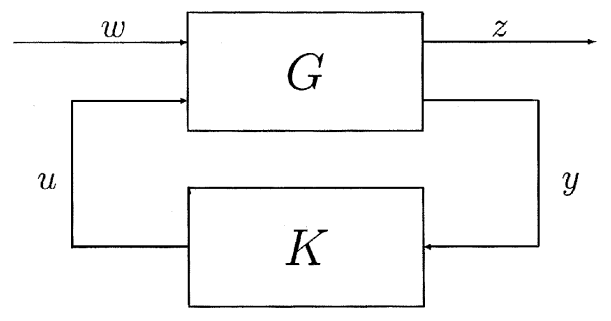

Fig. 1 Standard block diagram

system described by

$$
K=K_{O F} \triangleq \begin{aligned}
\hat{E}_{0} \dot{\hat{x}}_{0} & =\hat{A}_{0} \hat{x}_{0}+\hat{B}_{0} y \\
u & =\hat{C}_{0} \hat{x}_{0}
\end{aligned}
$$

where $\hat{x}_{0}$ is the state of the controller. Note that the plant and the controller are assumed the same structure but they may have different $E$ matrices. The objective of the control is to internally stabilize $G$ such that the closed-loop transfer function $T_{z w}$ is extended strictly positive real (ESPR, see section III for a precise definition). Here closed-loop internal stability means that the closed-loop system is regular, impulsefree, and that the states of $G$ and $K$ go to zero from all initial values when $w=0$.

This problem, referred to as the ESPR output feedback control problem, has been recently addressed and extensively studied in Sun et al. (1994) for linear time-invariant (LTI) plant and controller in state space model, in which several necessary and sufficient conditions in terms of solutions to algebraic Riccati equations or inequalities(ARE or ARI) were proposed for the solvability of the ESPR control problem. State-Space formulas for the controller design were also given in Sun et al. (1994). Most recently, Yung (1999) has characterized all solutions to this problem. He also adopted state space formulation.

The most general motivation for studying this problem stems from robust and nonlinear control: If the system uncertainties (linear and/or nonlinear) can be characterized by positive real properties, then the classical results in stability theory can be used to guarantee robust stability provided an appropriate closed-loop system is strictly positive real(Popov, 1973; Zames, 1966a, 1966b). Other materials come from circuit theory (Anderson, 1973) adaptive control (Astrom, 1983) and stability analysis (Narendra, 1973; Popov, 1973). It is beyond the scope of this paper to review the vast literature associated with positive realness theory. For a more extensive bibliography and review of the literature, see, e.g., Anderson et al. (1973), Joshi (1989), Narendra et al. (1973), Safonov et al. (1987), Sun et al. (1994), and the references cited therein.
The main point of this paper is that we adopt the descriptor systems model rather than the state space model. The control theory based on descriptor system models has been widely developed for many years: Cobb (1983) first gave a necessary and sufficient condition for the existence of an optimal solution to the linear quadratic optimization problem and also extensively studied the notions of controllability, observability and duality in descriptor systems (Cobb, 1984). The notions concerning controllability and observability for descriptor systems will not be reproduced here. See, e.g., Armentano (1986), Cobb (1984) for details. The generalized algebraic Riccati equations(GARE) for descriptor systems have been comprehensively studied during the past few years as well. Lewis (1986), Bender et al. (1987) and Takaba et al. (1994) constructed different kinds of GAREs for solving linear quadratic regulator problems based on certain assumptions. Recently, Kawamoto et al. (1996) elaborated the relationship between dissipation inequality and GARE. The problem of inconsistency of initial conditions was addressed there as well.

This paper is organized as follows: In section II, we investigate some properties of GAREs and GARIs. In section III, the ESPR output feedback control problems are examined. The derivations involve only elementary ideas beginning with a change of variables and a version of Positive Real Lemma for descriptor systems, thus the proofs given are simple. The Generalized Positive Real Lemma is also elaborated in detail in section III. Finally, some concluding remarks will be given in section IV.

The following notations will be used in this paper, throughout. Let $G$ in Fig. 1 be partitioned as

$$
G=\left[\begin{array}{ll}
G_{11} & G_{12} \\
G_{21} & G_{22}
\end{array}\right]
$$

then the transfer function $T_{z w}$ is expressed as the linear fractional transformation denoted by

$$
T_{z w}=L F T(G, K) \stackrel{\Delta}{=} G_{11}+G_{12} K\left(I-G_{22} K\right)^{-1} G_{21} .
$$

A compact packed matrix notation

$$
\left\{E,\left[\frac{A \mid B}{C} \mid D\right]\right\} \triangleq
$$

is used as well. See also Takaba et al. (1994).

\section{GENERALIZED ALGEBRAIC RICCATI EQUATIONS}

In this section we study the coupled generalized 
algebraic Riccati equation(GARE, Takaba et al. 1994)

$$
\left\{\begin{array}{l}
A^{T} X+X^{T} A+Q+X^{T} R X=0, \\
E^{T} X=X^{T} E \geq 0
\end{array}\right.
$$

and the generalized algebraic Riccati inequality (GARI)

$$
\left\{\begin{array}{l}
A^{T} P+P^{T} A+Q+P^{T} R P<0, \\
E^{T} P=P^{T} E \geq 0
\end{array}\right.
$$

together with the associated matrix pencil, called the Hamiltonian pencil,

$$
s\left[\begin{array}{cc}
E & 0 \\
0 & E^{T}
\end{array}\right]-\left[\begin{array}{cc}
A & R \\
-Q & -A^{T}
\end{array}\right]
$$

where $A, Q, R \in I R^{n \times n}$ with $Q, R$ symmetric. This pencil was named Hamiltonian since the matrix

$$
\bar{H} \triangleq\left[\begin{array}{cc}
A & R \\
-Q & -A^{T}
\end{array}\right]
$$

is a Hamiltonian matrix. Consider the following relatively simple descriptor system.

$$
\begin{aligned}
& E \dot{x}=A x+B u, \\
& y=C x+D u, \\
& E x\left(0^{-}\right)=E x_{0}, \quad G(\mathrm{~s}) \stackrel{\Delta}{=} D+C(s E-A)^{-1} B .
\end{aligned}
$$

It is well known that a descriptor system contains three different modes: finite dynamic modes, impulsive modes and nondynamic modes. For a detailed definition, see (Bender and Laub, 1987). Briefly, let $q \stackrel{\Delta}{=} \operatorname{deg} \operatorname{det}(s E-A)$. Then $\{E, A\}$ has $q$ finite dynamic modes, $r-q$ impulsive modes and $n-r$ nondynamic modes. Furthermore, if $r=q$, then there exist no impulsive modes and in this case the system is said to be impulse-free. $\{E, A\}$ is called stable if there exist no finite dynamic modes in $\operatorname{Re}[s] \geq 0 . \quad\{E, A\}$ is admissible if $\{E, A\}$ is regular, impulse-free and stable. The triple $\{E, A, B\}$ is said to be finite dynamics stabilizable and impulse controllable if there exists a constant matrix $K$ such that $\{E, A+B K\}$ is admissible. Similarly, $\{E, A, C\}$ is called finite dynamics detectable and impulse observable if there exists a constant matrix $L$ such that $\{E, A+L C\}$ is admissible. Without loss of generality, we can assume that the system (6) has a Weierstrass form:

$$
\begin{aligned}
& E=\left[\begin{array}{cc}
I & 0 \\
0 & N
\end{array}\right], A=\left[\begin{array}{cc}
A_{1} & 0 \\
0 & I
\end{array}\right], B=\left[\begin{array}{l}
B_{1} \\
B_{2}
\end{array}\right], \\
& \text { and } C=\left[\begin{array}{ll}
C_{1} & C_{2}
\end{array}\right] .
\end{aligned}
$$

where $N$ is a nilpotent matrix (that is, $N^{k}=0$ for some positive integer $k$ ).

Next, we introduce some important preliminary results, which are collected in the following and were essentially taken from Wang, Y. Y. et al. (1993), Takaba et al. (1994), and Wang, H. S. et al. (1998). Those results are slightly different from the existing version, but the proofs are pretty similar.

Proposition 1. Given a matrix quadruple $\{E, A, Q$, $R$, where $E, A, Q, R \in I^{n \times n}$ with symmetric $Q, R$, and two matrix pencils,

$$
P_{1}(s) \stackrel{\Delta}{=} s\left[\begin{array}{c}
-E \\
0
\end{array}\right]+\left[\begin{array}{l}
A \\
Q
\end{array}\right], \quad P_{2}(s) \stackrel{\Delta}{=} s\left[\begin{array}{ll}
-E & 0
\end{array}\right]+\left[\begin{array}{ll}
A & R
\end{array}\right] .
$$

Suppose that the pencil $s E-A$ is regular. Then we have the following:

(i) Suppose that $P_{1}(s)=0$ and $P_{2}(s)=0$ has no zeros in $\mathfrak{R e}(s) \geq 0$, namely $P_{1}(s)$ and $P_{2}(s)$ have full column and full row ranks, respectively, in $\mathfrak{R} e(s) \geq 0$. Suppose that $R \leq 0$. Furthermore suppose that $\{E, A, B\}$ is finite dynamic stabilizable and impulse controllable, and $\{E, A, C\}$ is finite dynamic detectable and impulse observable. Then the GARE (3) has an admissible solution $X_{E}$ with $E^{T} X_{E}=X_{E}^{T} E \geq 0$.

(ii) Consider (6) and the following GARE

$$
\left\{\begin{array}{l}
A^{T} X+X^{T} A+Q+\left(X^{T} B+C^{T} D\right) R\left(B^{T} X+D^{T} C\right)=0, \\
E^{T} X=X^{T} E \geq 0 .
\end{array}\right.
$$

Suppose that $Q, R \geq 0$. Then GARE (8) has an admissible solution $X_{E}$ with $E^{T} X_{E}=X_{E}^{T} E \geq 0$ if and only if $\Im m E+A(\aleph(E))=I R^{n}$ with $s E-A=0$ having no zeros in $\mathfrak{R} e(s) \geq 0$ and $G(s) \stackrel{\Delta}{=} C(s E-A)^{-1} B$ where $C^{T} C=Q$ and $I-$ $D^{T} D=R$ such that $\|G(\mathrm{~s})\|_{\infty}<1$. Here $\mathfrak{I} m E$ and $\aleph(E)$ denote the image and null space of $E$, respectively.

Recall that a solution to the GARE is said to be admissible if the pair $\{E, A+R X\}$ is admissible, i.e. stable and impulse-free. In view of Proposition 1, a corollary is readily obtained.

Corollary 2. Suppose that $Q \geq 0$ and $R \geq($ or $\leq) 0$. Suppose that $\{E, A, B\}$ is finite dynamic stabilizable and impulse controllable, $\{E, A, C\}$ is finite dynamic detectable and impulse observable, and $s E-A$ is regular. Furthermore, suppose that the Hamiltonian pencil (5) has no pure imaginary zeros and satisfies 


$$
\mathfrak{I} m \bar{E}+\bar{H}(\aleph(\bar{E}))=I R^{2 n}
$$

where $\bar{E} \triangleq\left[\begin{array}{cc}E & 0 \\ 0 & E^{T}\end{array}\right]$. Then the GARE (3) has an admissible solution.

In the sequel, we seek the condition in which one can deduce the existence of admissible solutions to GARE (3) from the existence of solutions to GARI (4). As we can see from the standard results on $H_{\infty}$ control for linear time-invariant systems, this deduction is of particular interest. In the subsequent development, we suppose that $\mathfrak{I} m E+A(\boldsymbol{\aleph}(E))=I R^{n}$. Consequently, we can assume, without loss of generality, that

$$
\{E, A\}=\left\{\left[\begin{array}{ll}
I & 0 \\
0 & 0
\end{array}\right],\left[\begin{array}{cc}
A_{1} & 0 \\
0 & -I
\end{array}\right]\right\}
$$

which is a modified Weierstrass form for impulsefree pair.

Lemma 3. Suppose that $\mathfrak{I} m E+A(\boldsymbol{N}(E))=I R^{n}$. Suppose that $R \geq 0$ and the pencil $P_{2}(s)$ has full row rank on the imaginary axis. Furthermore, suppose that GARI (4) has a nonsingular solution $P$ with $E^{T} P=P^{T} E \geq 0$ and is such that if $\{E, A\}$ is given in the form (9), $P$ can be chosen in the following form:

$$
P=\left[\begin{array}{cc}
P_{11} & 0 \\
P_{21} & P_{22}
\end{array}\right] \text { and } P_{11}>0, P_{22}>0 .
$$

Then the Hamiltonian pencil (5) has no pure imaginary zeros and satisfies

$$
\Im m \bar{E}+\bar{H}(\aleph(\bar{E}))=I R^{2 n}
$$

Proof. Set $Q=\left[\begin{array}{ll}Q_{11} & Q_{12} \\ Q_{21} & Q_{22}\end{array}\right]$ and $R=B B^{T}=$
$\left[\begin{array}{cc}B_{1} B_{1}^{T} & B_{1} B_{2}^{T} \\ B_{2} B_{1}^{T} & B_{2} B_{2}^{T}\end{array}\right]$ where the partition is compatible with form (9), and set

$$
S=A^{T} P+P^{T} A+Q+P^{T} B B^{T} P=\left[\begin{array}{ll}
S_{11} & S_{12} \\
S_{21} & S_{22}
\end{array}\right]
$$

Then $S<0$, by hypothesis. We first show that the Hamiltonian pencil is column-reduced. This is equivalent to showing that

$$
H_{22} \triangleq\left[\begin{array}{cc}
-I & B_{2} B_{2}^{T} \\
-Q_{22} & I
\end{array}\right]
$$

is nonsingular. Observe now that

$$
\begin{aligned}
S_{22} & =-P_{22}-P_{22}+Q_{22}+P_{22} B_{2} B_{2}^{T} P_{22} \\
& =(-I)^{T} P_{22}+P_{22}(-I)+Q_{22}+P_{22} B_{2} B_{2}^{T} P_{22}<0
\end{aligned}
$$

and clearly, $\left[\begin{array}{lll}I-j \omega & R_{22}\end{array}\right]$ has full row rank for all $\omega \in \mathbb{R}$. Then, by standard results of ARI (Algebraic Riccati Inequality), this implies that (11) has no eigenvalues on the $j \omega$-axis (see Knobloch et al., 1993), i.e. $H_{22}$ is nonsingular.

We can now show that the Hamiltonian pencil has no pure imaginary zeros. We assume, for convenience, that all signals may be complex (i.e. $C^{n}$ ) at this time. Observe the following identity

$$
\begin{aligned}
& x^{*}\left(A^{T} P+P^{T}\right) x+x^{*} Q x-u^{*} u+x^{*} \mathrm{P}^{T} B u+u^{*} B^{T} P x \\
& =x^{*}\left(A^{T} P+P^{T} A+Q+P^{T} B B^{T} P\right) x-\left(u-B^{T} P\right)^{*}\left(u-B^{T} P\right) \\
& =x^{*} S x-\left(u-B^{T} P\right)^{*}\left(u-B^{T} P\right) \leq x^{*} S x .
\end{aligned}
$$

Consider the descriptor system

$$
E \dot{x}=A x+B u
$$

Choose an input $u(\bullet)$, an initial condition $\operatorname{Ex}(0) \in C^{n}$ and let $x(\bullet)$ denote the corresponding solution. $\mathrm{Ob}$ serving that

$$
\frac{d\left(x^{*}(t) P^{T} E x(t)\right)}{d t}=x^{*}\left(A^{T} P+P^{T}\right) x+x^{*} P^{T} B u+u^{*} B^{T} P x,
$$

the relation (12) yields

$$
\frac{d\left(x^{*}(t) P^{T} E x(t)\right)}{d t}+x^{*} Q x-u^{*} u \leq x^{*} S x .
$$

Suppose, by contradiction, the pencil had zeros on the imaginary axis. By definition, there exist vectors $x_{0} \in C^{n}, p_{0} \in C^{n}$ and a number $\omega_{0} \in I R$ such that

$$
\left[\begin{array}{cc}
A-j \omega_{0} E & R \\
-Q & -A^{T}-j \omega_{0} E^{T}
\end{array}\right]\left[\begin{array}{l}
x_{0} \\
p_{0}
\end{array}\right]=\left[\begin{array}{l}
0 \\
0
\end{array}\right] .
$$

In this relation $x_{0} \neq 0$ because, if this were not the case, the identities

$$
\begin{aligned}
& R p_{0}=0 \\
& \left(A_{T}+j \omega_{0} E^{T}\right) p_{0}=0
\end{aligned}
$$

would contradict the hypothesis on pencil $P_{2}(s)$. The previous relation yields 


$$
\begin{aligned}
& p_{0}^{*}\left(A-j \omega_{0} E\right) x_{0}+p_{0}^{*} R p_{0}=0, \\
& x_{0}^{*} Q x_{0}+x_{0}^{*}\left(A-j \omega_{0} E\right)^{*} p_{0}=0,
\end{aligned}
$$

and therefore

$$
x_{0}^{*} Q x_{0}-p_{0}^{*} R p_{0}=0 .
$$

Set $u(t)=B^{T} p_{0} e^{j \omega_{0} t}$ and note that $E x(t)=E x_{0} e^{j \omega_{0} t}$ is the solution satisfying $\operatorname{Ex}(0)=E x_{0}$. Then

$$
\begin{aligned}
& x^{*}(t) P^{T} E x(t)=x_{0}^{*} P^{T} E x_{0}, \\
& x^{*}(t) Q x(t)-u^{*}(t) u(t)=x_{0}^{*} Q x_{0}-p_{0}^{*} R p_{0}=0, \\
& x^{*}(t) S x(t)=x_{0}^{*} S x_{0} .
\end{aligned}
$$

Inequality (13) yields $x_{0}^{*} S x_{0} \geq 0$ which is a contradiction, because $S$ is negative definite and $x_{0} \neq 0$. Q.E.D.

Lemma 4. Suppose that $\mathfrak{I} m E+A(\boldsymbol{N}(E))=I R^{n}$. Suppose that $Q \geq 0$ and the pencil $P_{1}(s)$ has full column rank on the imaginary axis. Furthermore, suppose that GARI (4) has a nonsingular solution $P$ with $E^{T} P=P^{T} E \geq 0$ and is such that if $\{E, A\}$ is given in the form (9), $P$ can be chosen in the following form:

$$
P=\left[\begin{array}{cc}
P_{11} & 0 \\
P_{21} & P_{22}
\end{array}\right] \text { and } P_{11}>0, P_{22}>0 .
$$

Then there exists an admissible solution $X_{E}$ to the GARE (3) with $E^{T} X_{E}=X_{E}^{T} E \geq 0$, and having the property that $\left\{E, A+R X_{E}\right\}$ is admissible.

Proof. The hypothesis on GARI (4) implies that

$$
s\left[\begin{array}{cc}
E^{T} & 0 \\
0 & E
\end{array}\right]-\left[\begin{array}{cc}
A^{T} & Q \\
-R & -A
\end{array}\right]=0
$$

has no zeros on the $j \omega$-axis and is column-reduced which, in turn, implies that

$$
s\left[\begin{array}{cc}
E & 0 \\
0 & E^{T}
\end{array}\right]-\left[\begin{array}{cc}
A & R \\
-Q & -A^{T}
\end{array}\right]=0
$$

has no zeros on the $j \omega$-axis and is column-reduced. Set

$$
Q=\left[\begin{array}{ll}
Q_{11} & Q_{12} \\
Q_{21} & Q_{22}
\end{array}\right] \text { and } R=\left[\begin{array}{ll}
R_{11} & R_{12} \\
R_{21} & R_{22}
\end{array}\right]
$$

where the partition is compatible with form (9). From
(4), there exists a positive definite matrix $P_{22}$ such that

$$
(-I) P_{22}+P_{22}(-I)+Q_{22}+P_{22}^{T} R_{22} P_{22}<0 .
$$

Clearly $\left[\begin{array}{ll}I-j \omega I & Q_{22}\end{array}\right]^{T}$ has full column rank for all $\omega \in \mathbb{R}$. This, again from the standard results of ARI (Knobloch et al., 1993) implies that there exists a matrix $X_{22}=X_{22}^{T} \geq 0$ satisfying the ARE (Algebraic Riccati Equation)

$$
-X_{22}-X_{22}+Q_{22}+X_{22} R_{22} X_{22}=0
$$

Rewrite pencil (14) as

$$
\begin{aligned}
& s\left[\begin{array}{cccc}
I & 0 & 0 & 0 \\
0 & I & 0 & 0 \\
\hline 0 & 0 & 0 & 0 \\
0 & 0 & 0 & 0
\end{array}\right]-\left[\begin{array}{cc|cc}
A_{1} & R_{11} & R_{12} & 0 \\
-Q_{11} & -A_{1}^{T} & 0 & -Q_{12} \\
\hline 0 & R_{12}^{T} & R_{22} & I \\
Q_{12}^{T} & 0 & I & Q_{22}
\end{array}\right] \\
& \Delta s\left[\begin{array}{cc}
\mathcal{T} & 0 \\
0 & 0
\end{array}\right]-\left[\begin{array}{cc}
\mathcal{T}_{1} & \mathcal{T}_{2} \\
\mathcal{T}_{3} & \mathcal{T}_{4}
\end{array}\right]
\end{aligned}
$$

From ARI (15), the above pencil can be simplified as

$$
s \mathcal{T}-\left[\mathcal{T}_{1}-\mathcal{T}_{2} \mathcal{T}_{4}^{-1} \mathcal{T}_{3}\right] \stackrel{\Delta}{=} s \mathcal{T}-\left[\begin{array}{cc}
\mathcal{A}_{0} & \mathcal{R}_{0} \\
-Q_{0} & -\mathcal{A}_{0}^{T}
\end{array}\right] .
$$

The existence of $P_{22}$ to ARI (15) implies that $Q_{0} \geq 0$ (See Willems 1971, Lemma 1). Moreover, GARI (4) and ARI (15) together imply that there exists a positive definite matrix $P_{0}\left(=P_{11}\right)$ satisfying

$$
S\left(P_{0}\right) \stackrel{\Delta}{=} \mathcal{A}_{0}^{T} P_{0}+P_{0} \mathcal{A}_{0}+Q_{0}+P_{0} \mathcal{R}_{0} P_{0}<0 .
$$

Equation (16) as well as the hypothesis on pencil $P_{2}(s)$ implies that there exists a stabilizing solution $X_{0} \geq 0$ satisfying the ARE $S\left(X_{0}\right)=0$ since one can deduce that $\left[\mathcal{A}_{0}^{T}-j \omega I \quad Q_{0}\right]^{T}$ has full column rank for all $\omega \in \mathbb{R}$ from the assumption that $\left[\begin{array}{ll}A^{T}-j \omega E^{T} & Q]^{T} \text { has full }\end{array}\right.$ column rank on the $j \omega$-axis. Set

$$
X_{21}=L_{2}-X_{22} L_{1} \text { and } X_{E}=\left[\begin{array}{cc}
X_{0} & 0 \\
X_{21} & X_{22}
\end{array}\right],
$$

where

$$
\left[\begin{array}{l}
L_{1} \\
L_{2}
\end{array}\right] \triangleq-\mathcal{T}_{4}^{-1} \mathcal{T}_{3}\left[\begin{array}{c}
I \\
X_{0}
\end{array}\right]
$$

which will satisfy the GARE (3) with $\left\{E, A+R X_{E}\right\}$ admissible. This completes the proof. Q.E.D.

We end up this section by introducing another 
important lemma which relates the GARE to GARI (in the opposite direction from Lemma 4.)

Lemma 5. Let $X^{-}$be a solution of GARE (3) having the property that $\left\{E, A+R X^{-}\right\}$is admissible. Then there exists a nonsingular matrix $P$ satisfying the GARI (4) and having the property that $E^{T} P=P^{T} E \geq E^{T} X$.

Proof. See Wang, H.S. et al. (1998) for detail.

\section{POSITIVE REAL CONTROL PROBLEM}

\section{Positive Real Systems in Descriptor Formula- tion}

We start this section with various definitions of positive real systems which are essentially generalizations of the definitions given in Sun et al. (1994) for a system in state space model.

Definition 6. Consider the descriptor system (6). Denote by $G(s)$ its transfer function matrix. Then

(1) The system (6) is said to be positive real (PR) if $G(s)$ is analytic in $\Re e(s)>0$ and satisfies $G(s)+$ $G^{T}\left(s^{*}\right) \geq 0, \forall \Re e(s)>0$,

(2) The system (6) is strictly positive real (SPR) if $G(s)$ is analytic in $\Re e(s) \geq 0$ and satisfies $G(j \omega)$ $+G^{T}(-j \omega)>0, \forall \omega \in[0, \infty)$,

(3) The system (6) is extended strictly positive real (ESPR) if it is $S P R$ and $G(j \infty)+G^{T}(-j \infty)>0$.

The following lemma is a generalization of the well known Kalman-Yacubovich-Popov positive real lemma to the descriptor system case. It plays a crucial role in our later proofs. This lemma connects the ESPR property of descriptor systems with solutions of GARE and GARI.

\section{Lemma 7. Generalized Positive Real Lemma}

Consider the system (6). Suppose that $D+D^{T \Delta}=\Delta$ $>0$ and $p=m$. Then the following statements are equivalent.

(i) The pair $\{E, A\}$ is admissible and $G(s)$ is ESPR,

(ii) The pair $\{E, A\}$ is admissible and the Hamiltonian pencil $\hat{P}(S) \triangleq S \widehat{E}-\hat{H}$ with

$\hat{E} \triangleq\left[\begin{array}{cc}E & 0 \\ 0 & E^{T}\end{array}\right]$, and $\hat{H} \triangleq\left[\begin{array}{cc}A-B \Delta^{-1} C & B \Delta^{-1} B^{T} \\ -C^{T} \Delta^{-1} C & -A^{T}+C^{T} \Delta^{-1} B^{T}\end{array}\right]$,

has no pure imaginary zeros and satisfies

$$
\mathfrak{I} m \hat{E}+\hat{H}(\aleph(\hat{E}))=I R^{2 n},
$$

(iii) The GARE

$\left\{\begin{array}{l}A^{T} X+X^{T} A+\left(X^{T} B-C^{T}\right) \Delta^{-1}\left(X^{T} B-C^{T}\right)^{T}=0, \\ E^{T} X=X^{T} E\end{array}\right.$

has an admissible solution $X_{E}$ with $E^{T} X_{E}=X_{E}^{T} E \geq 0$,

(iv) The GARI

$\left\{\begin{array}{l}A^{T} P+P^{T} A+\left(P^{T} B-C^{T}\right) \Delta^{-1}\left(P^{T} B-C^{T}\right)^{T}<0, \\ E^{T} P=P^{T} E\end{array}\right.$

has a nonsingular solution $P$ with $E^{T} P=P^{T} E \geq 0$.

Proof. Note that the GARE (18) can be rewritten as:

$$
\left\{\begin{array}{l}
\left(A-B \Delta^{-1} C\right)^{T} X+X^{T}\left(A-B \Delta^{-1} C\right)+C^{T} \Delta^{-1} C \\
+X^{T} B \Delta^{-1} B^{T} X=0 \\
E^{T} P=P^{T} E
\end{array}\right.
$$

Then the following implications are immediate from our preliminary results in section II.

$$
\text { (ii) } \Rightarrow \text { (iii) } \Rightarrow \text { (iv) }
$$

The equivalence between (i) and (iv) follows by using Propostion 1 and the fact that $G(s)$ is ESPR if and only if $\left\|\Delta^{\frac{1}{2}}+\Delta^{-\frac{1}{2}}\left(C-B^{T} P\right)(s E-A)^{-1} B\right\|_{\infty}<1$. It remains to show the implication: (i) $\Rightarrow$ (ii). First observe that

$\operatorname{det}[s \hat{E}-\hat{H}]=k \operatorname{det}[s E-A] \operatorname{det}\left[s E^{T}+A^{T}\right] \operatorname{det}\left[G^{T}(-s)+G(s)\right]$

where $k$ is some real constant. Clearly $\operatorname{det}[s \hat{E}-\hat{H}]=0$, by hypothesis, has no pure imaginary zeros and is not identical to zero. This implies that $\hat{P}(s)=0$ has no pure imaginary zeros. Next, since the pair $\{E, A\}$ is admissible, the two polynomials $\operatorname{det}[s E-A]$ and $\operatorname{det}\left[s E^{T}+A^{T}\right]$ both have degree $r$. Furthermore, since $G(s)$ is ESPR, the rational function $\operatorname{det}\left[G^{T}(-s)+G(s)\right]$ has relative degree 0 . These amount to showing that the polynomial $\operatorname{det}[s \hat{E}-\hat{H}]$ has degree $2 r$. This, in turn, implies that the pair $\{\hat{E}, \hat{H}\}$ is impulse-free, i.e., (17) is satisfied. This completes the proof. Q.E.D.

\section{ESPR Control Problem - State Feedback Case}

In this subsection, we first study a special and 
important case, namely the state feedback control. Consider the standard system connection shown in Fig. 1 with the plant $G$ given by

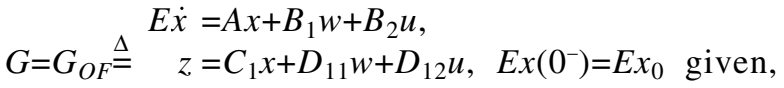

$$
\begin{aligned}
& y=x \text {. }
\end{aligned}
$$

The following standing assumptions are made throughout this paper.

(A1) $\left(D_{11}+D_{11}^{T}\right) \triangleq \mathrm{R}_{0}>0$,

(A2) $\left\{E, A, B_{2}\right\}$ is finite dynamic stabilizable and impulse controllable,

(A3) The matrix $D_{12}$ has full column rank

(A4) The matrix pencil $P_{1}(s) \stackrel{\Delta}{s} s E_{1}+H_{1}$, where

$$
E_{1}=\left[\begin{array}{cc}
-E & 0 \\
0 & 0
\end{array}\right], H_{1}=\left[\begin{array}{cc}
A & B_{2} \\
C_{1} & D_{12}
\end{array}\right]
$$

is of full column rank on the $j \omega$-axis and is column reduced (i.e., $\left.H_{1}^{-1}\left(\Im m E_{1}\right) \cap \boldsymbol{N}\left(E_{1}\right)=\{0\}\right)$.

(A5) $\left\{E, A, C_{2}\right\}$ is finite dynamic detectable and impulse observable

(A6) The matrix $D_{21}$ has full row rank,

(A7) The matrix pencil $P_{2}(s) \stackrel{\Delta}{=} s E_{2}+H_{2}$, where

$$
E_{2}=\left[\begin{array}{cc}
-E & 0 \\
0 & 0
\end{array}\right], H_{2}=\left[\begin{array}{cc}
A & B_{1} \\
C_{2} & D_{21}
\end{array}\right]
$$

is of full row rank on the $j \omega$-axis and is row reduced (i.e., $\left.\mathfrak{I} m E_{2}+H_{2}\left(\boldsymbol{\aleph}\left(E_{2}\right)\right)=I R^{n+p}\right)$. Note that $E_{1}$ is, in general, not equal to $E_{2}$ (They may have different sizes).

Before getting started, let's make some further observation on the ESPR control problem. Consider the standard system connection (1). Suppose that the following GARE

$R_{1}(X) \triangleq\left[A-B_{1} R_{0}^{-1} C_{1}-\left(B_{2}-B_{1} R_{0}^{-1} D_{12}\right) \Delta_{1}^{-1} D_{12}^{T} R_{0}^{-1} C_{1}\right]^{T} X$

$+X^{T}\left[A-B_{1} R_{0}^{-1} C_{1}-\left(B_{2}-B_{1} R_{0}^{-1} D_{12}\right) \Delta_{1}^{-1} D_{12}^{T} R_{0}^{-1} C_{1}\right]$

$+X^{T}\left[B_{1} R_{0}^{-1} B_{1}^{T}-\left(B_{2}-B_{1} R_{0}^{-1} D_{12}\right) \Delta_{1}^{-1}\left(B_{2}-B_{1} R_{0}^{-1} D_{12}\right)^{T}\right] X$

$+C_{1}^{T} R_{0}^{-1}\left(R_{0}-D_{12} \Delta_{1}^{-1} D_{12}^{T}\right) R_{0}^{-1} C_{1}=0 \quad E^{T} X=X^{T} E$

has an admissible solution $X_{E}$ with $E^{T} X_{E}=X_{E}^{T} E \geq 0$, where $\Delta_{1} \stackrel{\Delta}{=} D_{12}^{T} R_{0}^{-1} D_{12}$. Define a change of variables as follows (see also Yung,1999):

$$
\begin{aligned}
& r \stackrel{\Delta}{=} w-F_{1} x \\
& q \stackrel{\Delta}{=} D_{11} r+D_{12}\left(u-F_{2} x\right)
\end{aligned}
$$

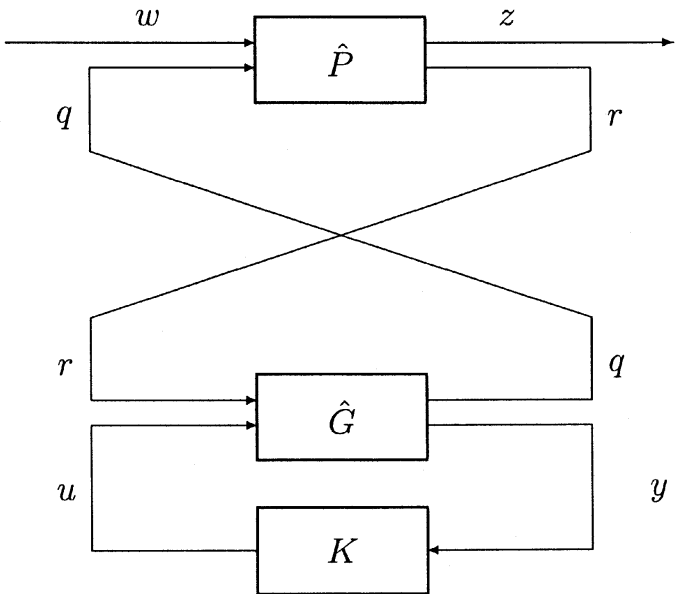

Fig. 2 Decomposition of the closed-loop system

Then

$$
q=-\left(D_{11} F_{1}+D_{12} F_{2}\right) x+D_{11} w+D_{12} u .
$$

where

$$
\begin{aligned}
& F_{2} \triangleq \Delta_{1}^{-1} D_{12}^{T} R_{0}^{-1}\left(B_{1}^{T} X_{E}-C_{1}\right)-\Delta_{1}^{-1} B_{2}^{T} X_{E} \\
& F_{1} \triangleq R_{0}^{-1}\left(B_{1}^{T} X_{E}-C_{1}-D_{12} F_{2}\right)
\end{aligned}
$$

Pre-multiplying (22) by $D_{12}^{T} R_{0}^{-1}$ and solving it for $u$, we obtain

$$
\begin{aligned}
u= & \left(F_{2}+\Delta_{1}^{-1} D_{12}^{T} R_{0}^{-1} D_{11} F_{1}\right) x-\Delta_{1}^{-1} D_{12}^{T} R_{0}^{-1} D_{11} w \\
& +\Delta_{1}^{-1} D_{12}^{T} R_{0}^{-1} q .
\end{aligned}
$$

Then the closed-loop system in Fig. 1 can be decomposed as two interconnected subsystems as follows:

$$
\begin{aligned}
& \begin{aligned}
E \dot{x}= & \left(A+B_{2} F_{2}+B_{2} \Delta_{1}^{-1} D_{12}^{T} R_{0}^{-1} D_{11} F_{1}\right) x \\
& \quad+\left(B_{1}-B_{2} \Delta_{1}^{-1} D_{12}^{T} R_{0}^{-1} D_{11}\right) w+B_{2} \Delta_{1}^{-1} D_{12}^{T} R_{0}^{-1} q
\end{aligned} \\
& z=\left(C_{1}+D_{11} F_{1}+D_{12} F_{2}\right) x+q \\
& r=-F_{1} x+w
\end{aligned}
$$

and

$$
\begin{aligned}
& E \dot{x}=\left(A+B_{1} F_{1}\right) x+B_{1} r+B_{2} u \\
& q=-D_{12} F_{2} x+D_{11} r+D_{12} u \\
& y=\left(C_{1}+D_{21} F_{1}\right) x+D_{21} w .
\end{aligned}
$$

This is shown pictorially in Fig. 2, where 


$$
\hat{P}=\left\{E,\left[\begin{array}{c|cc}
A+B_{2} F_{2} & B_{1}-B_{2} \Delta_{1}^{-1} D_{12}^{T} R_{0}^{-1} D_{11} & B_{2} \Delta_{1}^{-1} D_{12}^{T} R_{0}^{-1} \\
+B_{2} \Delta_{1}^{-1} D_{12}^{T} R_{0}^{-1} D_{11} F_{1} & & \\
\hline C_{1}+D_{11} F_{1}+D_{12} F_{2} & 0 & 0 \\
-F_{1} & I
\end{array}\right]\right\}
$$

and

$$
\hat{G}=\left\{E,\left[\begin{array}{c|cc}
A+B_{1} F_{1} & B_{1} & B_{2} \\
\hline-D_{12} F_{2} & D_{11} & D_{12} \\
C_{2}+D_{21} F_{1} & D_{21} & 0
\end{array}\right]\right\} .
$$

Let $x$ denote the state of $G$ with respect to a given input $w$, and $z$ its corresponding output. Then, by a standard game-theoretic argument, it can be shown that

$$
\begin{aligned}
& \frac{d}{d t}\left(x^{T} E^{T} X_{E} x\right)-z^{T} w \\
& =\frac{1}{2} x^{T} R_{1}\left(X_{E}\right) x-\frac{1}{2}\left[\begin{array}{l}
w-w^{*}(x) \\
u-u^{*}(x)
\end{array}\right]^{T} \mathcal{M}\left[\begin{array}{l}
w-w^{*}(x) \\
u-u^{*}(x)
\end{array}\right],
\end{aligned}
$$

where $u^{*}(x)=F_{2} x, w^{*}(x)=F_{1} x$ and

$$
\mathcal{M}=\left[\begin{array}{cc}
R_{0} & R_{12} \\
D_{12}^{T} & 0
\end{array}\right]
$$

Integrating (26) from zero to infinity and setting $x(0)$ $=x(\infty)=0$ yields

$$
\int_{0}^{\infty} z^{T}(t) w(t) d t=\int_{0}^{\infty} q^{T}(t) r(t) d t
$$

Equation (27) shows that $T_{z w}$ (transfer function matrix from $w$ to $z$ ) is ESPR if and only if $T_{q r}$ is. This is summarized in the following statement.

Proposition 8. Consider the standard system connection (1). Suppose that the following GARE

$R_{1}(X) \stackrel{\Delta}{=}\left[A-B_{1} R_{0}^{-1} C_{1}-\left(B_{2}-B_{1} R_{0}^{-1} D_{12}\right) \Delta_{1}^{-1} D_{12}^{T} R_{0}^{-1} C_{1}\right]^{T} X$

$+X^{T}\left[A-B_{1} R_{0}^{-1} C_{1}-\left(B_{2}-B_{1} R_{0}^{-1} D_{12}\right) \Delta_{1}^{-1} D_{12}^{T} R_{0}^{-1} C_{1}\right]$

$+X^{T}\left[B_{1} R_{0}^{-1} B_{1}^{T}-\left(B_{2}-B_{1} R_{0}^{-1} D_{12}\right) \Delta_{1}^{-1}\left(B_{2}-B_{1} R_{0}^{-1} D_{12}\right)^{T}\right] X$

$+C_{1}^{T} R_{0}^{-1}\left(R_{0}-D_{12} \Delta_{1}^{-1} D_{12}^{T}\right) R_{0}^{-1} C_{1}=0, \quad E^{T} X=X^{T} E$ has an admissible solution $X_{E}$ with $E^{T} X_{E}=X_{E}^{T} E \geq 0$. Then $K$ internally stabilizes $G$ and $T_{z w}$ is ESPR if and only if $K$ internally stabilizes $\hat{G}$ and $T_{q r}$ is ESPR.

The main result of this section reads as follows.

Theorem 9. Consider the plant $G=G_{S F}$ and assume that assumptions (A1) to (A4) are satisfied. Then there exists a state feedback controller $u=F_{x}$ such that the resulting closed-loop system is internally stable and ESPR if and only if the GARE

$$
\begin{aligned}
& R_{1}(X) \stackrel{\Delta}{=}\left[A-B_{1} R_{0}^{-1} C_{1}-\left(B_{2}-B_{1} R_{0}^{-1} D_{12}\right) \Delta_{1}^{-1} D_{12}^{T} R_{0}^{-1} C_{1}\right]^{T} X \\
& +X^{T}\left[A-B_{1} R_{0}^{-1} C_{1}-\left(B_{2}-B_{1} R_{0}^{-1} D_{12}\right) \Delta_{1}^{-1} D_{12}^{T} R_{0}^{-1} C_{1}\right] \\
& +X^{T}\left[B_{1} R_{0}^{-1} B_{1}^{T}-\left(B_{2}-B_{1} R_{0}^{-1} D_{12}\right) \Delta_{1}^{-1}\left(B_{2}-B_{1} R_{0}^{-1} D_{12}\right)^{T}\right] X \\
& +C_{1}^{T} R_{0}^{-1}\left(R_{0}-D_{12} \Delta_{1}^{-1} D_{12}^{T}\right) R_{0}^{-1} C_{1}=0, \quad E^{T} X=X^{T} E
\end{aligned}
$$

has an admissible solution $X_{E}$ with $E^{T} X_{E}=X_{E}^{T} E \geq 0$, where $\Delta_{1}=D_{12}^{T} R_{0}^{-1} D_{12}$. Moreover, when this condition holds, all ESPR state feedback controllers can be parameterized as $K=\operatorname{LFT}\left(M_{S F}, Q\right)$, where

$$
M_{S F}=\left\{E,\left[\begin{array}{c|cc}
A+B_{1} F_{1}+B_{2} F_{2} & 0 & B_{2} \\
\hline 0 & F_{2} & I \\
-I & I & 0
\end{array}\right]\right\},
$$

$Q \in R \mathcal{H}^{\circ}$, and $D_{11}+D_{12} Q P$ is ESPR with

$$
P=\left\{E,\left[\begin{array}{c|c}
A+B_{1} F_{1}+B_{2} F_{2} & B_{1} \\
\hline I & 0
\end{array}\right]\right\},
$$

in which

$$
\begin{aligned}
& F_{2} \triangleq \Delta_{1}^{-1} D_{12}^{T} R_{0}^{-1}\left(B_{1}^{T} X_{E}-C_{1}\right)-\Delta_{1}^{-1} B_{2}^{T} X_{E} \\
& F_{1} \triangleq R_{0}^{-1}\left(B_{1}^{T} X_{E}-C_{1}-D_{12} F_{2}\right)
\end{aligned}
$$




\section{(i) Proof of Necessity}

Suppose that $u=F x$ is one such controller. Using this as a feedback law and closing the loop to get (see Fig. 1)

$$
T_{z w}=\left\{E,\left[\begin{array}{c|c}
A+B_{2} F & B_{1} \\
\hline C_{1}+D_{12} F & D_{11}
\end{array}\right]\right\}
$$

which is internally stable and ESPR. By generalized Positive Real Lemma (Lemma 7), it follows that there exists a nonsingular matrix $P$ satisfying the GARI

$$
\left\{\begin{array}{l}
\left(A+B_{2} F\right)^{T} P+P^{T}\left(A+B_{2} F\right)+\left[P^{T} B_{1}\right. \\
\left.\quad-\left(C_{1}+D_{12} F\right)^{T}\right] R_{0}^{-1}\left[P^{T} B_{1}-\left(C_{1}+D_{12} F\right)^{T}\right]^{T}<0, \\
E^{T} P=P^{T} E \geq 0 .
\end{array}\right.
$$

After some algebraic manipulation, the above GARI can be rewritten in a more comapct form, i.e.

$$
R_{1}(P)<0 \text {, with } E^{T} P=P^{T} E \geq 0 \text {. }
$$

We see that if the hypotheses in Lemma 4 were satisfied, then we can deduce from (30) that GARE $R_{1}(X)=0$ has an admissible solution. In view of Lemma 4, we need to prove the following:

1. The pencil

$$
\left[\begin{array}{l}
-j \omega E+\left[A-B_{1} R_{0}^{-1} C_{1}-\left(B_{2}-B_{1} R_{0}^{-1} D_{12}\right) \Delta_{1}^{-1} D_{12}^{T} R_{0}^{-1} C_{1}\right] \\
C_{1}^{T} R_{0}^{-1}\left(R_{0}-D_{12} \Delta_{1}^{-1} D_{12}^{T}\right) R_{0}^{-1} C_{1}
\end{array}\right]
$$

has no pure imaginary zeros.

It is easy to verify this point by assumption (A4).

2. $\left\{E, A-B_{1} R_{0}^{-1} C_{1}-\left(B_{2}-B_{1} R_{0}^{-1} D_{12}\right) \Delta_{1}^{-1} D_{12}^{T} R_{0}^{-1} C_{1}\right\}$ is impulse-free.

Observe the following identity,

$$
\begin{gathered}
=\left[\begin{array}{cc}
I & -\left(B_{2}-B_{1} R_{0}^{-1} D_{12}\right) \Delta_{1}^{-1} D_{12}^{T} R_{0}^{-1} \\
0 & I
\end{array}\right]\left[\begin{array}{cc}
I & -B_{1} R_{0}^{-1} \\
0 & I
\end{array}\right] \\
\cdot\left[\begin{array}{cc}
-j \omega E+A & B_{2} \\
C_{1} & D_{12}
\end{array}\right]
\end{gathered}
$$

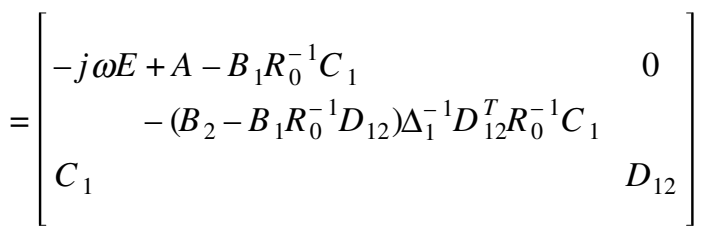

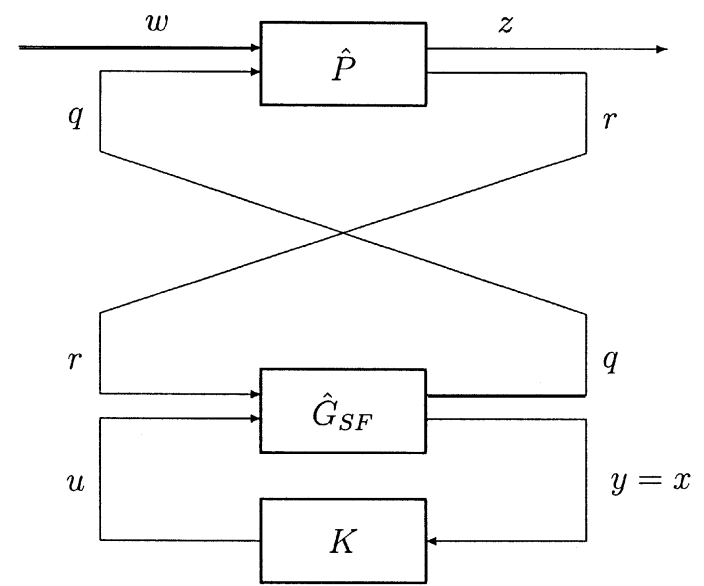

Fig. 3 Decomposition of the closed-loop system in state feedback case

Since $P_{1}(s)$ is column reduced, the matrix pencil on the right side of the above identity should be column reduced as well. This implies that $\{E, A-$ $\left.B_{1} R_{0}^{-1} C_{1}-\left(B_{2}-B_{1} R_{0}^{-1} D_{12}\right) \Delta_{1}^{-1} D_{12}^{T} R_{0}^{-1} C_{1}\right\}$ is impulse-free. This completes the proof of necessity.

\section{(ii) Proof of Sufficiency}

Suppose now that the GARE $R_{1}(X)=0$ has an admissible solution $X_{E}$. Motivated by Proposition 8, we first make a change of variables defined as (21) which we reproduce here for the sake of clarity.

$$
\begin{aligned}
& r \stackrel{\Delta}{=} w-F_{1} x \\
& q \stackrel{\Delta}{=} D_{11} r+D_{12}\left(u-F_{2} x\right) .
\end{aligned}
$$

Then the closed-loop system in Fig. 1 with $G=G_{S F}$ can be decomposed as two interconnected subsystems as follows:

$$
\begin{aligned}
& E \dot{x}=\left(A+B_{2} F_{2}+B_{2} \Delta_{1}^{-1} D_{12}^{T} R_{0}^{-1} D_{11} F_{1}\right) x \\
& +\left(B_{1}-B_{2} \Delta_{1}^{-1} D_{12}^{T} R_{0}^{-1} D_{11}\right) w+B_{2} \Delta_{1}^{-1} D_{12}^{T} R_{0}^{-1} q \\
& z=\left(C_{1}+D_{11} F_{1}+D_{12} F_{2}\right) x+q \\
& r=-F_{1} x+w
\end{aligned}
$$

and

$$
\begin{aligned}
& E \dot{x}=\left(A+B_{1} F_{1}\right) x+B_{1} r+B_{2} u \\
& q=-D_{12} F_{2} x+D_{11} r+D_{12} u \\
& y=x .
\end{aligned}
$$

This is shown pictorially in Fig. 3, where 


$$
\hat{P}=\left\{E,\left[\begin{array}{c|cc}
A+B_{2} F_{2} & B_{1}-B_{2} \Delta_{1}^{-1} D_{12}^{T} B_{0}^{-1} D_{11} & B_{2} \Delta_{1}^{-1} D_{12}^{T} R_{0}^{-1} \\
+B_{2} \Delta_{1}^{-1} D_{12}^{T} R_{0}^{-1} D_{11} F_{1} & & I \\
\hline C_{1}+D_{11} F_{1}+D_{12} F_{2} & 0 & 0 \\
-F_{1} & I
\end{array}\right]\right\}
$$

and

$$
\hat{G}_{S F}=\left\{E,\left[\begin{array}{c|cc}
A+B_{1} F_{1} & B_{1} & B_{2} \\
\hline-D_{12} F_{2} & D_{11} & D_{12} \\
I & 0 & 0
\end{array}\right]\right\}
$$

Set $K=\left\{E_{K},\left[\begin{array}{c|c}A_{K} \mid B_{K} \\ \hline C_{K} \mid\end{array}\right]\right\}$. Then the dynamical equations for the linear fractional transformation $\operatorname{LFT}\left(\hat{G}_{S F}, K\right)$ are:

$$
\begin{aligned}
\operatorname{LET}\left(\hat{G}_{S F}, K\right) & =\left\{\left[\begin{array}{cc}
E & 0 \\
0 & E_{K}
\end{array}\right],\left[\begin{array}{cc|c}
A+B_{1} F_{1} & B_{2} C_{k} & B_{1} \\
B_{K} & A_{K} & 0 \\
\hline-D_{12} F_{2} & D_{12} C_{K} & D_{11}
\end{array}\right]\right\} \\
& \triangleq\left\{\hat{E},\left[\begin{array}{c}
\hat{A} \mid \hat{B} \\
\hat{C} \mid \hat{D}
\end{array}\right],\right.
\end{aligned}
$$

and the dynamical equations for the closed-loop system $\operatorname{LFT}\left(\mathrm{G}_{S F}, K\right)$ are

$$
\begin{aligned}
\operatorname{LFT}\left(G_{S F}, K\right) & =\operatorname{LFT}\left(\hat{P}, \operatorname{LFT}\left(\hat{G}_{S F}, K\right)\right) \\
& \triangleq\left\{E_{C},\left[\frac{A_{C} \mid B_{C}}{C_{C} D_{C}}\right]\right\}
\end{aligned}
$$

where

$$
A_{C}=\left[\begin{array}{cc}
A+B_{2} F_{2} & A_{C 1} \\
A_{C 2} & \hat{A}
\end{array}\right]
$$

with $A_{C 1}=\left[\begin{array}{ll}-B_{2} F_{2} & B_{2} C_{K}\end{array}\right]$ and $A_{C 2}=\left[\begin{array}{c}-B_{1} F_{1} \\ 0\end{array}\right]$,

$$
B_{C}=\left[\begin{array}{c}
B_{1} \\
\hat{B}
\end{array}\right], \quad C_{C}=\left[C_{1}+D_{12} F_{2} \hat{C}\right],
$$

and $D_{C}=D_{11}, E_{C}=\left[\begin{array}{cc}E & 0 \\ 0 & \hat{E}\end{array}\right]$

Let $\widehat{\Delta} \triangleq \hat{D}^{T}+\hat{D}$ and $\Delta_{C} \triangleq D_{C}^{T}+D_{C}$. It is easy to see that $\hat{\Delta}=D_{C}=R_{0}>0$. Now using equations Eqs. (28) and (29), it is straightforward but tedious to verify that, with

$$
X_{C} \triangleq\left[\begin{array}{cc}
X_{E} & 0 \\
0 & \hat{X}
\end{array}\right]
$$

for some $\hat{X}$,

$$
\begin{gathered}
R_{C}\left(X_{C}\right) \stackrel{\Delta}{=}\left(A_{C}-B_{C} \Delta_{C}^{-1} C_{C}\right)^{T} X_{C}+X_{C}^{T}\left(A_{C}-B_{C} \Delta_{C}^{-1} C_{C}\right) \\
+C_{C}^{T} \Delta_{C}^{-1} C_{C}+X_{C}^{T} B_{C} \Delta_{C}^{-1} B_{C}^{T} X_{C} \\
=\left[\begin{array}{cc}
R_{1}\left(X_{E}\right) & 0 \\
0 & \hat{R}(\hat{X})
\end{array}\right]
\end{gathered}
$$

where

$$
\begin{aligned}
\hat{R}(\hat{X}) \triangleq & \left(\hat{A}-\hat{B} \hat{\Delta}^{-1} \hat{C}\right)^{T} \hat{X}+\hat{X}^{T}\left(\hat{A}-\hat{B} \hat{\Delta}^{-1} \hat{C}\right) \\
& +\hat{C}^{T} \hat{\Delta}^{-1} \hat{C}+\hat{X}^{T} \hat{B} \hat{\Delta}^{-1} \hat{B}^{T} \hat{X}
\end{aligned}
$$

Furthermore, it is straightforward to verify that

$$
\begin{aligned}
& A_{C}-B_{C} \Delta_{C}^{-1} C_{C}+B_{C} \Delta_{C}^{-1} B_{C}^{T} X_{C} \\
& =\left[\begin{array}{cc}
A+B_{1} F_{1}+B_{2} F_{2} & * \\
0 & \hat{A}-\hat{B} \hat{\Delta}^{-1} \hat{C}+\hat{B} \hat{\Delta}^{-1} \hat{B}^{T} \hat{X}
\end{array}\right]
\end{aligned}
$$

where the '*' stands for an irrelevant entry. Hence, using the fact that $X_{E}$ is an admissible solution to the GARE $R_{1}(X)=0$ together with Proposition 8, we can conclude that $X_{C}$ is an admissible solution to the GARE $R_{C}\left(X_{C}\right)=0$ if and only if $\hat{X}$ is an admissible solution to the GARE $\hat{R}(\hat{X})=0$, and, in addition, $E_{C}^{T} X_{C}=X_{C}^{T} E_{C} \geq 0$ if $\hat{E}^{T} \hat{X}=\hat{X}^{T} \hat{E} \geq 0$. Then $K$ internally stabilizes $G_{S F}$ and $T_{z w}$ is ESPR if $K$ internally stabilizes $\hat{G}_{S F}$ and $T_{q r}$ is ESPR. The rest of the proof is 
then to find all internally stabilizing $K$ for $\widehat{G}_{S F}$ such that $T_{q r}$ is ESPR.

Take an admissible realization of $Q$ :

$$
Q=\left\{E_{Q},\left[\frac{A_{Q} \mid B_{Q}}{C_{Q} \mid D_{Q}}\right]\right\} .
$$

Since $Q \in R \mathcal{H}^{\infty},\left\{E_{Q}, A_{Q}\right\}$ is admissible. Note that the dynamical equations for $D_{11}+D_{12} Q P$ are

$$
\begin{aligned}
& \left\{\left[\begin{array}{cc}
E & 0 \\
0 & E_{Q}
\end{array}\right],\left[\begin{array}{cc|c}
A+B_{1} F_{1}+B_{2} F_{2} & 0 & B_{1} \\
B_{Q} & A_{Q} & 0 \\
\hline D_{12} D_{Q} & D_{12} C_{Q} & D_{11}
\end{array}\right]\right\} \\
& \triangleq\left\{\bar{E},\left[\frac{\bar{A}}{\bar{C}} \mid \frac{\bar{B}}{\bar{D}}\right]\right\} .
\end{aligned}
$$

The fact that $\left\{E, A+B_{1} F_{1}+B_{2} \mathrm{~F}_{2}\right\}$ is admissible implies that $\{\bar{E}, \bar{A}\}$ is also admissible. Together with the hypothesis that $D_{11}+D_{12} Q P$, is ESPR, it follows from Lemma 7 that there exists a matrix $\bar{X}$ which satisfies the GARE

$$
\left\{\begin{array}{l}
\left(\bar{A}-\bar{B} R_{0}^{-1} \bar{C}\right)^{T} \bar{X}+\bar{X} \bar{X}^{T}\left(\bar{A}-\bar{B} R_{0}^{-1} \bar{C}\right) \\
\quad+\bar{C} R_{0}^{-1} \bar{C}+\bar{X} \bar{C}^{T} R_{0}^{-1} \bar{B} T \bar{X}=0 \\
\bar{E} T \bar{X}=\bar{X}^{T} \bar{E}
\end{array}\right.
$$

with $\bar{E}^{T} \bar{X}=\bar{X}^{T} \bar{E}>0$ and is such that the matrix pair $\left\{\bar{E}, \bar{A}-\bar{B} R_{0}^{-1} \bar{C}+\bar{B} R_{0}^{-1} \frac{B}{T} \bar{X}\right\}$ is admissible. The dynamical equations for the closed-loop system are:

$\operatorname{LFT}\left(G_{S F}, K\right)=\operatorname{LFT}\left(G_{S F}, \operatorname{LFT}\left(M_{S F}, Q\right)\right)$

$=\left\{\begin{array}{ccc}E & 0 & 0 \\ 0 & E & 0 \\ 0 & 0 & E_{Q}\end{array}\right]$,

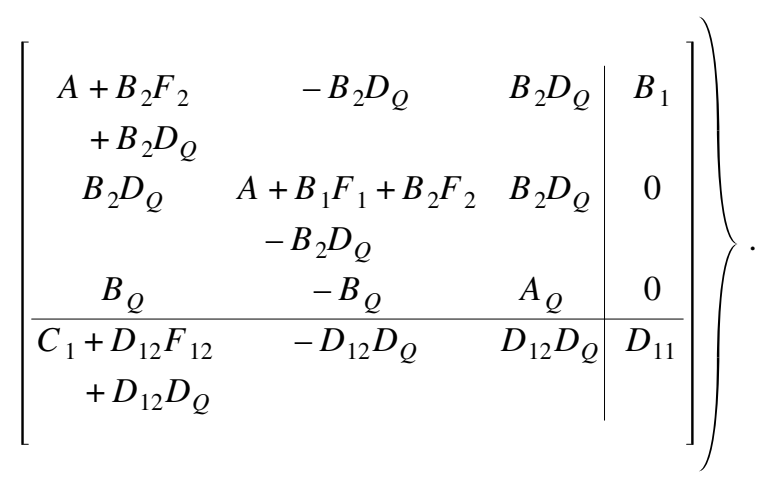

Apply an equivalent transformation with $M=$ $\left[\begin{array}{ccc}I & 0 & 0 \\ I & -I & 0 \\ 0 & 0 & I\end{array}\right]$ on the left and $N=M^{-1}$ on the right to the last realization to get

$$
\operatorname{LFT}\left(G_{S F}, K\right)=\left\{E^{\circ},\left[\frac{A^{\circ} \mid B^{\circ}}{C^{\circ} \mid D^{\circ}}\right]\right\},
$$

where

$$
\begin{aligned}
E^{\circ} & =\left[\begin{array}{ccc}
E & 0 & 0 \\
0 & E & 0 \\
0 & 0 & E_{Q}
\end{array}\right] \\
A^{\circ} & =\left[\begin{array}{cc}
A+B_{2} F_{2} & A_{1}^{\circ} \\
A_{2}^{\circ} & \bar{A}
\end{array}\right]
\end{aligned}
$$

with

$$
\begin{aligned}
& A_{1}^{\circ}=\left[\begin{array}{ll}
B_{2} D_{Q} & B_{2} D_{Q}
\end{array}\right] \text { and } A_{2}^{\circ}=\left[\begin{array}{c}
-B_{1} F_{1} \\
0
\end{array}\right], \\
& B^{\circ}=\left[\frac{B_{1}}{B}\right], \quad C^{\circ}=\left[C_{1}+D_{12} F_{2} \bar{C}\right]
\end{aligned}
$$

and

$$
D^{\circ}=\bar{D}=D_{11}
$$

Then it can be shown by a routine calculation that the matrix

$$
X^{\circ} \stackrel{\Delta}{=}\left[\begin{array}{cc}
X_{E} & 0 \\
0 & \bar{X}
\end{array}\right]
$$

satisfies the GARE

$$
\left\{\begin{array}{l}
\left(A^{\circ}-B^{\circ} R_{0}^{-1} C^{\circ}\right)^{T} X^{\circ}+X^{\circ} T\left(A^{\circ}-B^{\circ} R_{0}^{-1} C^{\circ}\right) \\
\quad+C^{\circ} T R_{0}^{-1} C^{\circ}+X^{\circ} T B^{\circ} R_{0}^{-1} B^{\circ} X^{\circ}=0, \\
E^{\circ} T X^{\circ}=X^{\circ} T E^{\circ},
\end{array}\right.
$$

with $E^{\circ T} X^{\circ}=X^{\circ T} E^{\circ} \geq 0$. Moreover, it is straightforward to show that the matrix pair

$$
\begin{aligned}
& \left\{E^{\circ}, A-B^{\circ} R_{0}^{-1} C^{\circ}+B^{\circ} R_{0}^{-1} B^{\circ} X^{\circ}\right\} \\
& =\left\{\left[\begin{array}{cc}
E & 0 \\
0 & E
\end{array}\right],\left[\begin{array}{cc}
A+B_{1} F_{1} & * \\
+B_{2} F_{2} & \\
0 & \bar{A}-\bar{B} R_{0}^{-1} \bar{C} \\
& +\bar{B} R_{0}^{-1} \bar{B} T \bar{X}
\end{array}\right]\right\}
\end{aligned}
$$


is admissible. Hence, $X^{\circ}$ is an admissible solution to the GARE (32). It now follows from Generalized Positive Real lemma that the controller $K=L F T\left(M_{S F}\right.$, $Q$ ) for any given $Q \in \mathcal{R} \mathcal{H}^{\circ}$ with $D_{11}+D_{12} Q P$ ESPR does internally stabilize $G_{S F}$ and render the closed-loop system ESPR.

To complete the proof, we need to show that for any given state feedback controller $\hat{K}$ that achieves closed-loop internal stability and ESPR can be expressed in the form of $\operatorname{LFT}\left(M_{S F}, \hat{Q}\right)$ for some $\hat{Q} \in R \mathcal{H}^{\circ}$ with $D_{11}+D_{12} \hat{Q} P$ ESPR. Now introduce

$$
\hat{M}_{S F} \triangleq\left\{E,\left[\begin{array}{c|cc}
A+B_{1} F_{1} & -B_{2} F_{2} & B_{2} \\
\hline-F_{2} & -F_{2} & I \\
I & I & 0
\end{array}\right]\right\}
$$

Then $\hat{K}$ internally stabilizes $\hat{M}_{S F}$ since $\hat{K}$ internally stabilizes $\hat{G} S F$ by Proposition 8. Thus $\operatorname{LFT}\left(\hat{M}_{S F}, \hat{K}\right)$ $\in \mathcal{R} \mathcal{H}^{\infty}$. Let $\hat{Q} \stackrel{\Delta}{=} \operatorname{LFT}\left(\hat{M}_{S F}, \hat{K}\right) \in \mathcal{R} \mathcal{H}^{\infty}$. Then it can be shown after some algebraic manipulation that $D_{11}+D_{12} \hat{Q} P=L F T\left(\widehat{G}_{S F}, \hat{K}\right)$. Again, it follows from Proposition 8 that $D_{11}+D_{12} \hat{Q} P$ is ESPR. Moreover, it is straightforward to verify that $\operatorname{LFT}\left(M_{S F}, \hat{Q}\right)=L F T$ $\left(M_{S F}, \operatorname{LFT}\left(\hat{M}_{S F}, \hat{K}\right)\right)=\operatorname{LFT}\left(N_{S F}, \hat{K}\right)$, where

$N_{S F}=$

$\left\{\left[\begin{array}{cc}E & 0 \\ 0 & E\end{array}\right],\left[\begin{array}{cc|cc}A+B_{1} F_{1}+2 B_{2} F_{2} & -B_{2} F_{2} & -B_{2} F_{2} & B_{2} \\ B_{2} F_{2} & A+B_{1} F_{1} & -B_{2} F_{2} & B_{2} \\ \hline F_{2} & -F_{2} & 0 & I \\ -I & I & I & 0\end{array}\right]\right\}$.

Conjugating the states of $N_{S F}$ by $\left[\begin{array}{cc}I & 0 \\ -I & I\end{array}\right]$ on the left and $\left[\begin{array}{cc}I & 0 \\ -I & I\end{array}\right]^{-1}=\left[\begin{array}{ll}I & 0 \\ I & I\end{array}\right]$ on the right yields

$$
\begin{aligned}
N_{S F} & =\left\{\left[\begin{array}{cc}
E & 0 \\
0 & E
\end{array}\right],\left[\begin{array}{cc|cc}
A+B_{1} F_{1} & -B_{2} F_{2} & -B_{2} F_{2} & B_{2} \\
+B_{2} F_{2} & & & \\
0 & A+B_{1} F_{1} & 0 & 0 \\
& +B_{2} F_{2} & & \\
\hline 0 & -F_{2} & 0 & I \\
0 & I & I & 0
\end{array}\right]\right\} \\
& =\left[\begin{array}{ll}
0 & I \\
I & 0
\end{array}\right] .
\end{aligned}
$$

Thus $\operatorname{LFT}\left(M_{S F}, \hat{Q}\right)=L F T\left(N_{S F}, \hat{K}\right)=\hat{K}$. This completes the proof.

Remark. The parameter $Q$ above is governed by an ESPR-like constraint. The weighting matrices $D_{12}$ and $\hat{P}$ can be eliminated by multiplying certain matrix inverses as described in Zhou (1996) which gives all state feedback controllers for $\mathcal{H}^{\circ}$ control problem. Nevertheless, the influence of $D_{11}$ cannot be eradicated.

\section{ESPR OUTPUT FEEDBACK CONTROL PROBLEM}

\section{Central Controller}

Before proceeding to the main problem of this paper, we need a preliminary result which is stated in the following theorem.

Theorem 10. Consider the standard system connection (Fig. 1) with

$$
\begin{gathered}
E \dot{x}=A x+B_{1} w+B_{2} u \\
G=G_{F I} \stackrel{\Delta}{=} z=C_{1} x+D_{11} w+D_{12} u, \\
y=\left[\begin{array}{l}
x \\
y
\end{array}\right] .
\end{gathered}
$$

Suppose that assumption (A1) to (A4) hold. Then the ESPR full information control problem for descriptor systems is solvable if and only if the GARE $R_{1}(X)=0$ has an admissible solution $X_{E}$ with $E^{T} X_{E}=$ $X_{E}^{T} E \geq 0$. Moreover, if this condition is satisfied, one such controller is given by $u=\left[\begin{array}{ll}F_{2} & 0\end{array}\right]$.

Remark. The proof is pretty similar to the proof of Theorem 9 and is, thus, omitted. Note that the assumption (A1) can be replaced by a weaker one. We will not pursue this point here.

The following theorem provides a the necessary and sufficient condition for the solvability of ESPR output feedback control problem.

Theorem 11. Consider the standard system connection (Fig. 1) with $G=G_{O F}$. Suppose that assumptions (A1) to (A7) hold. Then the following statements are equivalent.

(I) There exists a controller of the form (2) such that the resulting closed-loop system is internally stable and ESPR.

(II) (i) the GARE $R_{1}(X)=0$ has an admissible solution $X_{E}$ with $E^{T} X_{E}=X_{E}^{T} E \geq 0$.

(ii) the GARE 


$$
\begin{aligned}
R_{3}(Z) \stackrel{\Delta}{=} & A_{Z} Z+Z^{T} A_{Z}^{T}-Z^{T}\left(C_{2 Z}^{T} \Delta_{2}^{-1} C_{2 Z}-F_{2}^{T} \Delta_{1} F_{2}\right) Z \\
& +B_{1 Z} R_{0}^{-1} B_{1 Z}^{T}=0 \\
& E Z=Z^{T} E^{T}
\end{aligned}
$$

has an admissible solution $Z_{E}$ with $E Z_{E}=$ $Z_{E}^{T} E^{T} \geq 0$, where

$$
\begin{aligned}
& \Delta_{2} \stackrel{\Delta}{=} D_{21} R_{0}^{-1} D_{21}^{T} \\
& A_{Z} \triangleq A+B_{1} R_{0}^{-1}\left(B_{1}^{T} X_{E}-C_{1}\right)-B_{1} R_{0}^{-1} D_{21}^{T} \Delta_{2}^{-1} C_{2 Z} \\
& B_{1 Z} \stackrel{\Delta}{=} B_{1}\left(I-R_{0}^{-1} D_{21}^{T} \Delta_{2}^{-1} D_{21}\right) \\
& C_{2 Z} \triangleq C_{2}+D_{21} R_{0}^{-1}\left(B_{1}^{T} X_{E}-C_{1}\right)
\end{aligned}
$$

Moreover, when these conditions are satisfied, one such controller is given as in the form (2) with

$$
\begin{aligned}
\hat{E}_{0}= & E \\
\hat{A}_{0}= & A+B_{2} \hat{C}_{0}-\hat{B}_{0} C_{2} \\
& +\left(B_{1}-\hat{B}_{0} D_{21}\right) R_{0}^{-1}\left(B_{1}^{T} X_{E}-C_{1}-D_{12} \hat{C}_{0}\right) \\
\hat{B}_{0}= & \left(Z_{E}^{T} C_{2 Z}^{T}+B_{1} R_{0}^{-1} D_{21}^{T}\right) \Delta_{2}^{-1} \\
\hat{C}_{0}= & F_{2}
\end{aligned}
$$

Before giving the proof of the theorem, we note that the hierarchically coupled pair of GAREs given above can be further manipulated to yield two mutually decoupled GAREs, with a separated additional spectral radius condition. This is summarized in the following statement.

Corollary 12. Suppose that GARE $R_{1}(X)=0$ has an admissible $X_{E}$ with $E^{T} X_{E}=X_{E}^{T} E \geq 0$. Suppose also that the following conditions are satisfied.

\section{(i) The GARE}

$$
\begin{aligned}
& R_{2}(Y) \stackrel{\Delta}{=}\left[A-B_{1} R_{0}^{-1} C_{1}-B_{1} R_{0}^{-1} D_{21}^{T} \Delta_{2}^{-1}\left(C_{2}-D_{21} R_{0}^{-1} C_{1}\right)\right] Y \\
& +Y^{T}\left[A-B_{1} R_{0}^{-1} C_{1}-B_{1} R_{0}^{-1} D_{21}^{T} \Delta_{2}^{-1}\left(C_{2}-D_{21} R_{0}^{-1} C_{1}\right)\right]^{T} \\
& +Y^{T}\left[C_{1}^{T} R_{0}^{-1} C_{1}-\left(C_{2}-D_{21} R_{0}^{-1} C_{1}\right)^{T} \Delta_{2}^{-1}\left(C_{2}-D_{21} R_{0}^{-1} C_{1}\right)\right] Y \\
& +B_{1} R_{0}^{-1}\left(R_{0} R_{21}^{T} \Delta_{2}^{-1} D_{21}\right) R_{0}^{-1} B_{1}^{T}=0 \\
& \quad E Y=Y^{T} E^{T},
\end{aligned}
$$

has an admissible solution $Y_{E}$ with $E Y_{E}=Y_{E}^{T} E^{T} \geq 0$

(ii) The Spectral Radius $\rho\left(Y_{E}, X_{E}\right)<1$

Then the condition (II) of Theorem 11 holds.
Moreover, when these conditions are satisfied, the matrices $X_{E}, Y_{E}$ and $Z_{E}$ have the following relationship:

$$
Z_{E}=\left(I-Y_{E} X_{E}\right)^{-1} Y_{E}=Y_{E}\left(I-X_{E} Y_{E}\right)^{-1} .
$$

Remark. This corollary is easily verified by involving some simple algebraic calculations. Unlike the LTI case [7], this result is, in general, not a necessary and sufficient condition since the matrix $I+X_{E} Z_{E}$ may not be invertible.

\section{(i) Proof of Sufficiency of Theorem 11}

Observe that the closed-loop system (1)-(2) can be written as

$$
\begin{aligned}
& \varepsilon_{C}\left[\begin{array}{l}
\dot{x} \\
\dot{e}
\end{array}\right]=\mathcal{A}_{C}\left[\begin{array}{l}
x \\
e
\end{array}\right]+\mathcal{B}_{C} w \\
& z=C_{C}\left[\begin{array}{l}
x \\
e
\end{array}\right]+\mathcal{D}_{C} w
\end{aligned}
$$

where

$$
\begin{aligned}
& \mathcal{E}_{C} \triangleq\left[\begin{array}{cc}
E & 0 \\
0 & \hat{E}_{0}
\end{array}\right] \\
& \mathcal{A}_{C} \triangleq\left[\begin{array}{cc}
A+B_{2} \hat{C}_{0} & -B_{2} \hat{C}_{0} \\
A-\hat{A}_{0}+B_{2} \hat{C}_{0}-\hat{B}_{0} \hat{C}_{2} & \hat{A}_{0}-B_{2} \hat{C}_{0}
\end{array}\right] \\
& \mathcal{B}_{C} \triangleq\left[\begin{array}{c}
B_{1} \\
B_{1}-\hat{B}_{0} D_{21}
\end{array}\right] \\
& \mathcal{C}_{C} \triangleq\left[\begin{array}{ll}
C_{1}+D_{12} \hat{C}_{0} & -D_{12} \hat{C}_{0}
\end{array}\right] \\
& \mathcal{D}_{C} \triangleq D_{11}
\end{aligned}
$$

and $e \stackrel{\Delta}{=} x-\hat{x}_{0}$

The GARE $R_{3}(Z)=0$ can be rewritten as:

$$
\begin{aligned}
R_{3}(Z)= & \left(\bar{A}_{Z}-\bar{B}_{Z} R_{0}^{-1} \bar{C}_{Z}\right) Z+Z^{T}\left(\bar{A}_{Z}-\bar{B}_{Z} R_{0}^{-1} \bar{C}_{Z}\right)^{T} \\
& +\bar{B}_{Z} R_{0}^{-1} \bar{B}_{Z}^{T}+Z^{T} \bar{C}_{Z}^{T} R_{0}^{-1} \bar{C}_{Z} Z=0, \\
& E Z=Z^{T} E^{T}
\end{aligned}
$$

where

$$
\bar{A}_{Z} \triangleq A_{Z}-Z_{E}^{T} C_{2 Z}^{T} \Delta_{2}^{-1} C_{2 Z}+\bar{B}_{Z} R_{0}^{-1} \bar{C}_{Z}
$$

$$
\bar{B}_{Z} \triangleq B_{1 Z}-Z_{E}^{T} C_{2 z}^{T} \Delta_{2}^{-1} D_{21}
$$

$\bar{C}_{Z} \triangleq R_{0}^{1 / 2} \Delta_{1}^{1 / 2} F_{2}$ 
Note that $Z_{E}$ is also the admissible solution to GARE (33).

By Generalized Positive Real Lemma, we can conclude that $\left\{E^{T}, \bar{A}_{Z}^{T}\right\}$ is admissible and $\bar{B}_{Z}^{T}\left(s E^{T}-\right.$ $\left.\bar{A}_{Z}^{T}\right)^{-1} \bar{C}_{Z}^{T}+D_{11}^{T}$ is ESPR. This, in turn, implies that $\left\{E, \bar{A}_{Z}\right\}$ is admissible and $\bar{C}_{Z}\left(s E-\bar{A}_{Z}\right)^{-1} \bar{B}_{Z}+D_{11}$ is ESPR. GARE

Again by Generalized Positive Real Lemma, the

$$
\begin{aligned}
R_{4}(W)= & \left(\bar{A}_{Z}-\bar{B}_{Z} R_{0}^{-1} \bar{C}_{Z}\right)^{T} W+W^{T}\left(\bar{A}_{Z}-\bar{B}_{Z} R_{0}^{-1} \bar{C}_{Z}\right) \\
& +\bar{C}_{Z}^{T} R_{0}^{-1} \bar{C}_{Z}+W^{T} \bar{B}_{Z} R_{0}^{-1} \bar{B}_{Z}^{T} W=0 \\
& E^{T} W=W^{T} E
\end{aligned}
$$

has an admissible solution $W_{E}$ with $E^{T} W_{E}=W_{E}^{T} E \geq 0$.

Now Set

$$
\mathcal{P}_{C} \triangleq\left[\begin{array}{cc}
X_{E} & 0 \\
0 & W_{E}
\end{array}\right] .
$$

Clearly, $\mathcal{E}_{C}^{T} \mathcal{P}_{C}=\mathcal{P}_{C}^{T} \mathcal{E}_{C}$. Lengthy but otherwise routine calculation shows that $\mathscr{P}_{C}$ is an admissible solution to the GARE

$$
\begin{aligned}
& \mathcal{A}_{C}^{T} \mathcal{P}+\mathcal{P}^{T} \mathcal{A}_{C}+\left(\mathcal{P}^{T} \mathcal{B}_{C}-C_{C}^{T}\right) R_{0}^{-1}\left(\mathcal{P}^{T} \mathcal{B}_{C}-C_{C}^{T}\right)^{T}=0, \\
& \varepsilon_{C}^{T} \mathcal{P}=\mathcal{P}^{T} \varepsilon_{C}
\end{aligned}
$$

with $\varepsilon_{C}^{T} \mathcal{P}_{C}=\mathcal{P}_{C}^{T} \varepsilon_{C} \geq 0$. It follows, again from Generalized Positive Real Lemma, that (2) is a stabilizing controller such that $T_{z w}$ is ESPR. This completes the proof of sufficiency.

\section{(ii) Proof of Necessity of Theorem 11}

Suppose that $K_{O F}$ is one solution. Then the controller $K_{F I}=K_{O F}\left[\begin{array}{ll}C_{2} & D_{21}\end{array}\right]$ solves the FI problem. From Theorem 10, it follows that GARE $R_{1}(X)=0$ has an admissible solution. Consequently, we see that $F_{1}$, $F_{2}$ are well defined. As in the state feedback case, we shall make a change of variables described by (21). Then by a similar argument as in the proof of Theorem 9, the closed-loop system in Fig. 1 can be decomposed as two interconnected subsystems. This is illustrated in Fig. 4, where $\hat{P}$ is given as in (31) and

$$
\hat{G}_{O F}=\left\{E,\left[\begin{array}{c|cc}
A+B_{1} F_{1} & B_{1} & B_{2} \\
\hline-D_{12} F_{2} & D_{11} & D_{12} \\
C_{2}+D_{21} F_{1} & D_{21} & 0
\end{array}\right]\right\}
$$

Since the GARE $R_{1}(X)=0$ has an admissible

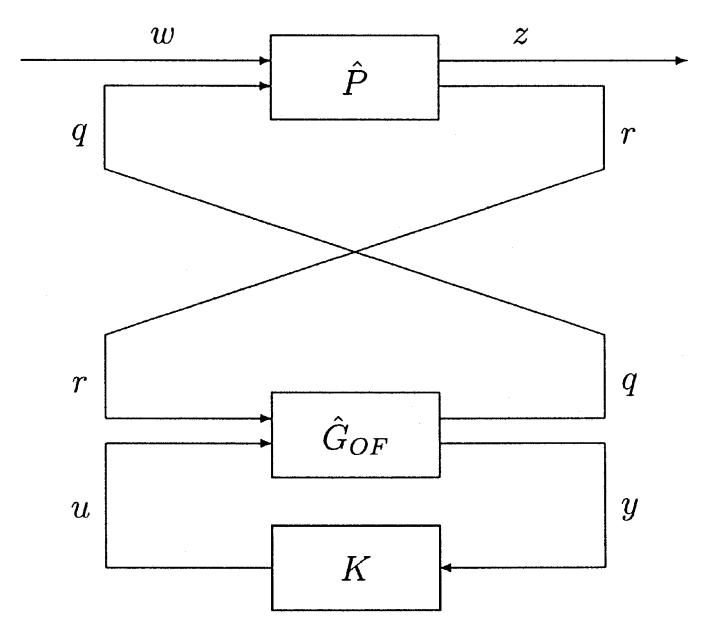

Fig. 4 Decomposition of the closed-loop system in output feedback case

solution $X_{E}$ with $E^{T} X_{E}=X_{E}^{T} E \geq 0$, then, by Proposition 8, $K$ internally stabilizes $G_{O F}$ and $T_{z w}$ is ESPR if and only if $K$ internally stabilizes $\hat{G}_{O F}$ and $T_{q r}$ is ESPR. The rest of the necessity proof follows by observing first that $K$ internally stabilizes $\hat{G}_{O F}$ and $T_{q r}$ is ESPR if and only if $K^{T}$ internally stabilizes $\hat{G}_{O F}^{T}$ and $T_{q r}^{T}$ is ESPR. Now introduce

$G_{b} \stackrel{\Delta}{=} \hat{G}_{O F}^{T}$

$$
=\left\{E^{T},\left[\begin{array}{c|cc}
\left(A+B_{1} F_{1}\right)^{T} & -F_{2}^{T} D_{12}^{T} & \left(C_{2}+D_{21} F_{1}\right)^{T} \\
\hline B_{1}^{T} & D_{11}^{T} & D_{21}^{T} \\
B_{2}^{T} & D_{12}^{T} & 0
\end{array}\right]\right\}
$$

$$
\triangleq\left\{\tilde{E},\left[\begin{array}{c|cc}
\tilde{A} & \tilde{B}_{1} & \tilde{B}_{2} \\
\hline \tilde{C}_{1} & \tilde{D}_{11} & \tilde{D}_{12} \\
\tilde{C}_{1} & \tilde{D}_{21} & 0
\end{array}\right]\right\}
$$

As a consequence of Proposition 8 and the above observation, the ESPR OF problem for $G_{b}$ is also solvable. Again, suppose that $K_{b}$ solves the ESPR OF problem for $G_{b}$ then the controller $K_{b}=\left[\begin{array}{ll}\tilde{C}_{2} & \tilde{D}_{21}\end{array}\right]$ solves the FI problem inherited from $G_{b}$. It follows, again from Theorem 10, that GARE

$$
\begin{aligned}
& \tilde{R}_{1}(X) \triangleq\left[\tilde{A}-\tilde{B}_{1} \tilde{R}_{0}^{-1} \tilde{C}_{1}-\left(\tilde{B}_{2}-\tilde{B}_{1} \tilde{R}_{0}^{-1} \tilde{D}_{12}\right) \tilde{\Delta}_{1}^{-1} \tilde{D}_{12}^{T} \tilde{R}_{0}^{-1} \tilde{C}_{1}\right]^{T} X \\
& +X^{T}\left[\tilde{A}-\tilde{B}_{1} \tilde{R}_{0}^{-1} \tilde{C}_{1}-\left(\tilde{B}_{2}-\tilde{B}_{1} \tilde{R}_{0}^{-1} \tilde{D}_{12}\right) \tilde{\Delta}_{1}^{-1} \tilde{D}_{12}^{T} \tilde{R}_{0}^{-1} \tilde{C}_{1}\right] \\
& +X\left[\tilde{B}_{1} \tilde{R}_{0}^{-1} \tilde{B}_{1}^{T}-\left(\tilde{B}_{2}-\tilde{B}_{1} \tilde{R}_{0}^{-1} \tilde{D}_{12}\right) \tilde{\Delta}_{1}^{-1}\left(\tilde{B}_{2}-\tilde{B}_{1} \tilde{R}_{0}^{-1} \tilde{D}_{12}\right)^{T}\right] X \\
& +\tilde{C}_{1}^{T} \tilde{R}_{0}^{-1}\left(\tilde{R}_{0}-\tilde{D}_{12} \tilde{\Delta}_{1}^{-1} \tilde{D}_{12}^{T}\right) \tilde{R}_{0}^{-1} \tilde{C}_{1}
\end{aligned}
$$




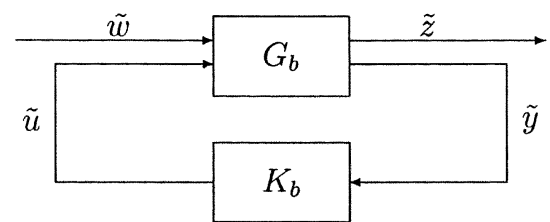

Fig. 5 Block diagram for $\operatorname{LFT}\left(G_{b}, K_{b}\right)$

$\tilde{E}^{T} X=X^{T} \tilde{E}$

has an admissible solution $\tilde{X}$ with $\tilde{E}^{T} \tilde{X}=\tilde{X}^{T} \tilde{E} \geq 0$ where $\tilde{R}_{0}=\tilde{D}_{11}^{T}+\tilde{D}_{11}=R_{0}$ and $\widetilde{\Delta}_{1}=\tilde{D}_{12}^{T} \tilde{R}_{0}^{-1} \tilde{D}_{12}=\Delta_{2}$. It is easy to verify that $\tilde{R}_{1}(X)$ is exactly $R_{3}(X)$. This shows that $\tilde{X} \stackrel{\Delta}{=} Z_{E}$ indeed exists. This completes the necessity proof.

\section{Characterization of All ESPR Output Feedback Controllers}

The following theorem, which is the main result of this section, characterizes all output feedback controllers that achieve closed-loop internal stability and ESPR

Theorem 13. Suppose that assumptions (A1) to (A7) are satisfied. Suppose also that condition (II) of Theorem 11 holds. Then the set of all output feedback controllers that achieve closed-loop internal stability and ESPR can be parameterized as $K=L F T$ $\left(M_{O F}, Q\right)$, where

$$
M_{O F}=\left\{E,\left[\begin{array}{c|cc}
\hat{A}_{0} & \hat{B}_{0} & \hat{B}_{1} \\
\hline \hat{C}_{0} & 0 & I \\
-\left(C_{2}+D_{21} F_{1}\right) & I & 0
\end{array}\right]\right\},
$$

$\widehat{A}_{0}, \hat{B}_{0}, \hat{C}_{0}$ are defined as in Theorem 11,

$$
\begin{aligned}
\hat{B}_{1} \triangleq & \left(I+X_{E} Z_{E}\right)^{T}\left(B_{2}-B_{1} R_{0}^{-1} D_{12}\right) \\
& +\left(C_{1} Z_{E}+D_{21}^{T} \hat{B}_{0}^{T}\right)^{T} R_{0}^{-1} D_{12}
\end{aligned}
$$

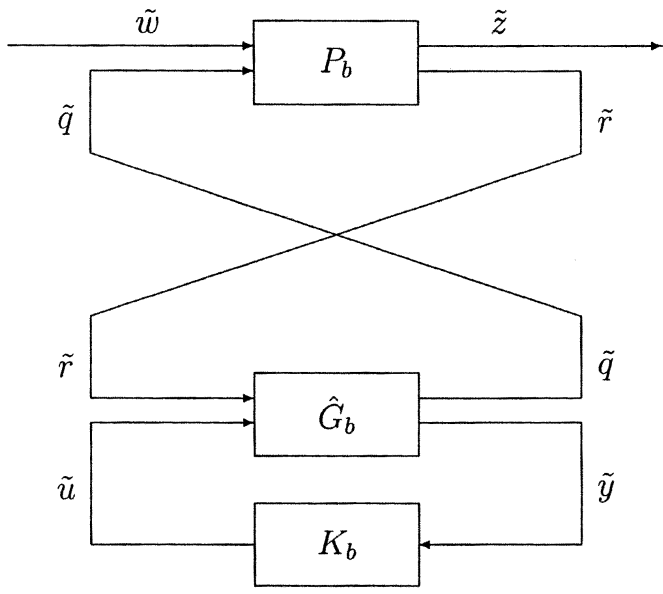

Fig. 6 Decomposition of $\operatorname{LFT}\left(G_{b}, K_{b}\right)$

and $Q \in R \mathcal{H}^{\circ}$ with $D_{11}+D_{12} Q D_{21}$ ESPR.

Proof. From subsection (ii), the problem of parameterizing all internally stabilizing controllers for $G_{O F}$ such that $T_{z w}$ is ESPR is equivalent to the problem of parameterizing all internally stabilizing controllers $K_{b}$ for $G_{b}$ such that $T_{q r}^{T}$ is ESPR. In what follows, we shall focus on the problem of parameterization of all internally stabilizing controllers $K_{b}$ for $G_{b}$ such that $T_{q r}^{T}$ is ESPR. Denote by $\tilde{x},\{\tilde{w}, \tilde{u}\}$ and $\{\tilde{z}, \tilde{y}\}$ the state, inputs and outputs of $G_{b}$, respectively, as shown in Fig. 5. Next, make a change of variables as follows:

$$
\begin{aligned}
& \tilde{r} \stackrel{\Delta}{=} \tilde{w}-\tilde{F}_{1} \tilde{x} \\
& \tilde{q} \stackrel{\Delta}{=} \tilde{D}_{11} \tilde{r}+\tilde{D}_{12}\left(\tilde{u}-\tilde{F}_{2} \tilde{x}\right)
\end{aligned}
$$

where

$$
\begin{aligned}
& \tilde{F}_{1} \stackrel{\Delta}{=} \tilde{R}_{0}^{-1}\left(\tilde{B}_{1}^{T} \tilde{X}-\tilde{C}_{1}-\tilde{D}_{12} \tilde{F}_{2}\right) \\
& \tilde{F}_{2} \stackrel{\Delta}{=} \widetilde{\Delta}_{1}^{-1} \tilde{D}_{12}^{T} \tilde{R}_{0}^{-1}\left(\tilde{B}_{1}^{T} \tilde{X}-\tilde{C}_{1}\right)-\tilde{\Delta}_{1}^{-1} \tilde{B}_{2}^{T} \tilde{X} .
\end{aligned}
$$

Then it follows, by direct verification using the same arguments as before, that the closed-loop system in Fig. 5 can be decomposed as two interconnected subsystems. This is shown pictorially in Fig. 6, where

$$
P_{b}=\left\{\tilde{E},\left[\begin{array}{c|cc}
\tilde{A}+\tilde{B}_{2} \tilde{F}_{2} & \tilde{B}_{1}-\tilde{B}_{2} \tilde{\Delta}_{1}^{-1} \tilde{D}_{12}^{T} \tilde{R}_{0}^{-1} \tilde{D}_{11} & \tilde{B}_{2} \tilde{\Delta}_{1}^{-1} \tilde{D}_{12}^{T} \tilde{R}_{0}^{-1} \\
+\tilde{B}_{2} \tilde{\Delta}_{1}^{-1} \tilde{D}_{12}^{T} \tilde{R}_{0}^{-1} \tilde{D}_{11} \tilde{F}_{1} & & \\
\hline \tilde{C}_{1}+\tilde{D}_{11} \tilde{F}_{1}+\tilde{D}_{12} \tilde{F}_{2} & 0 & I \\
\tilde{F}_{1} & I & 0
\end{array}\right]\right\}
$$


and

$$
\hat{G}_{b}=\left\{\tilde{E},\left[\begin{array}{c|cc}
\tilde{A}+\tilde{B}_{1} \tilde{F}_{1} & \tilde{B}_{1} & \tilde{B}_{2} \\
\hline-\tilde{D}_{12} \tilde{F}_{2} & \tilde{D}_{11} & \tilde{D}_{12} \\
\tilde{C}_{2}+\tilde{D}_{21} \tilde{F}_{1} & \tilde{D}_{21} & 0
\end{array}\right]\right\}
$$

Again, applying Proposition 8 shows that $K_{b}$ internally stabilizes $G_{b}$ and $T_{q r} T=T_{\tilde{z} \tilde{w}}$ is ESPR if and only if $K_{b}$ internally stabilizes $\widehat{G}_{b}$ and $T_{\tilde{q} \tilde{r}}$ is ESPR.

Now let $L=F_{2}^{T}=\hat{C}_{0}^{T}$, then simple algebra shows that $\left\{\tilde{E}, \tilde{A}+\tilde{B}_{1} \tilde{F}_{1}+L\left(\tilde{C}_{2}+\tilde{D}_{21} \tilde{F}_{1}\right)\right\}=\left\{E^{T},\left(A+B_{1} F_{1}+\right.\right.$ $\left.B_{2} F_{2}\right)^{T}$ which is admissible. Also note that if we let $F=-\hat{B}_{0}^{T}$, then it is straightforward to verify that $\{\tilde{E}$, $\left.\tilde{A}+\tilde{B}_{1} \tilde{F}_{1}+\tilde{B}_{2} F\right\}=\left\{E^{T}, A_{Z}^{T}-C_{2 Z}^{T} \Delta_{2}^{-1} C_{2 Z} Z_{E}+F_{2}^{T} \Delta_{1} F_{2} Z_{E}\right\}$, which is also admissible since $Z_{E}$ is an admissible solution to $R_{3}(Z)=0$. Then, from Theorem 2 of Wang, H. S. et al. (1997), all internally stabilizing controllers for $\hat{G}_{b}$ can be parameterized as $K_{b}=\operatorname{LFT}\left(M_{b}\right.$, $Q_{b}$ ) with $Q_{b} \in \mathcal{R} \mathcal{H}^{\circ}$, where

$$
M_{b}=\left\{\tilde{E},\left[\begin{array}{c|cc}
\tilde{A}+\tilde{B}_{1} \tilde{F}_{1}-\tilde{B}_{2} \hat{B}_{0}^{T} & -\hat{C}_{0}^{T} & \tilde{B}_{2} \\
+\hat{C}_{0}^{T}\left(\tilde{C}_{2}+\tilde{D}_{21} \tilde{F}_{1}\right) & & \\
\hline-\hat{B}_{0}^{T} & 0 & I \\
-\left(\tilde{C}_{2}+\tilde{D}_{21} \tilde{F}_{1}\right) & I & 0
\end{array}\right]\right\} .
$$

It is easy to see that $M_{b}$ can be rewritten as

$$
M_{b}=\left\{\tilde{E},\left[\begin{array}{c|cc}
\hat{A}_{0}^{T} & -\hat{C}_{0}^{T} & \left(C_{2}+D_{21} F_{1}\right)^{T} \\
\hline-\hat{B}_{0}^{T} & 0 & I \\
-\hat{B}_{1}^{T} & I & 0
\end{array}\right]\right\},
$$

where $\hat{B}_{1}$ is as defined by (34).

Moreover, a little bit of algebra shows that

$$
T_{\tilde{q} \tilde{r}}=\operatorname{LFT}\left(\hat{G}_{b}, \operatorname{LFT}\left(M_{b}, Q_{b}\right)\right)=D_{11}^{T}+D_{21}^{T} Q_{b} D_{12}^{T} .
$$

So $T_{\tilde{q} \tilde{r}}$ is ESPR if and only if $D_{11}^{T}+D_{21}^{T} Q_{b} D_{12}^{T}$ is ESPR.

Thus all internally stabilizing controllers for $\hat{G}_{O F}^{T}$ such that $T_{q r}^{T}$ is ESPR can be parameterized as $K_{b}=L F T\left(M_{b}, Q_{b}\right)$, where $Q_{b} \in \mathcal{R} \mathcal{H}^{\infty}$ with $D_{11}^{T}+$ $D_{21}^{T} Q_{b} D_{12}^{T}$ ESPR. Consequently, all internally stabilizing controllers for $\hat{G}_{O F}$ such that $T_{q r}$ is ESPR can be parameterized as $K=K_{b}^{T}=\operatorname{LFT}\left(M_{b}^{T}, Q\right)$ where $Q^{\Delta} Q_{b}^{T} \in \mathcal{R H}_{\mathcal{H}^{\circ}}$ with $D_{11}+D_{12} Q D_{21}$ ESPR. It is easy to see that $M_{b}^{T}=M_{O F}$. This completes the proof. Q.E.D.

The parametrization given above is an implicit one. We leave this form here for the readers' sake of comparision with the SF parametrizaton. However, the parametrization can be explicitly defined via simple algebraic manipulations.

Theorem 14. Suppose that assumptions (A1) to (A7) are satisfied. Suppose also that condition (II) of Theorem 11 holds. Then the set of all output feedback controllers that achieve closed-loop internal stability and ESPR can be parameterized as $K=L F T$ $\left(M_{b \text { OF }}^{T}, \hat{G}\right)$, where

$$
M_{O F}=\left\{\tilde{E},\left[\begin{array}{c|cc}
\hat{A}_{0}-\hat{B}_{1} D_{12}^{-L} & \hat{B}_{0}+\hat{B}_{1} D_{12}^{-L} & \hat{B}_{1} D_{12}^{-L} \\
\cdot D_{11}\left(D_{21}^{-R}+F_{1}\right) & \cdot D_{11} D_{21}^{-R} & \\
\hline-\hat{C}_{0}-D_{12}^{-L} D_{11} & D_{12}^{-L} D_{11} D_{21}^{-R} & D_{12}^{-L} \\
\cdot\left(D_{21}^{-R}+F_{1}\right) & & \\
-\left(D_{21}^{-R} C_{2}+F_{1}\right) & D_{21}^{-R} & 0
\end{array}\right]\right\} \text {, }
$$

with $\hat{Q}$ ESPR, where $D_{12}^{-L}$ and $D_{21}^{-R}$ denote any left and right inverses of $D_{12}$ and $D_{21}$, respectively.

Proof. The proof is fairly straightforward, thus omitted.

\section{CONCLUSION}

In this paper, we have given the descriptor formulations of all state feedback and output feedback controllers, respectively, that achieve closed-loop internal stability and ESPR. We have also investigated some properties of the GARE. The ESPR control problem for which $D_{11}+D_{11}^{T}>0$ (assumption (A1)) is examined. (A1) is a necessary condition for an ESPR problem to be solvable in the usual linear timeinvariant case; but, quite the contrary, this is no longer the case for the descriptor systems.

As a bottleneck of the quadratic optimization problems, we believe it is time for us to survey some properties of the generalized algebraic Riccati equation. In this paper, we have recovered some properties of the coupled GARE (3) and GARI (4). It's seen that GAREs have a property similar to the monotonicity of AREs: If $X$ is an admissible solution of a GARE and $P$ is a solution of the GARI deduced from the GARE, then, $x^{T} E^{T} P x-x^{T} E^{T} X x>0$ for all $x \in \mathfrak{I} m E$.

Parameterization of all output feedback controllers is via the Youla parameterization for descriptor systems. The parameterization is given in an explicit form; but this is not the situation for the state feedback parameterization. We see that state feedback controllers have no influence on " $D_{11} w$ " term; hence, it is reasonable that the constraint on $Q$ should involve the $D_{11}$ matrix. 


\section{NOMENCLATURE}

$\mathfrak{I} m E$
$\frac{\mathfrak{N}(E)}{C^{+}}$
$E, A, B, B_{1}, B_{2}, C, C_{1}, C_{2}$
$D, D_{12}, D_{21}$
$A_{k}, B_{1 k}, C_{1 k}, D_{k}, R$
$X, P$

$G(s), K(s)$

$J_{1}(u, w), J_{2}(u, w)$

$X_{1}, X_{2}, Q_{1}, Q_{2}$

$K, K_{1}, K_{2}$

$\mathcal{H}_{2}$

$\mathcal{H}_{\infty}$

$\mathcal{R} \mathcal{H}^{\infty}$ plane GARE trices image space of matrix $E$ null space of matrix $E$ closed right-half complex

constant matrices

constant matrices

constant matrices

variable matrix in generalized algebraic Riccati equation (GARE)

transfer function matrices cost functional used in Nash game admissible solution of

static feedback gain ma-

Hilbert space

Hardy space

the set of all real rational proper stable matrix functions

\section{REFERENCES}

1. Anderson, B.D.O., and Vongpanitlerd, S., 1973, Network Analysis and Synthesis -A Modern Systems Theory Approach. Prentice-Hall, Englewood Cliffs, N. J.

2. Armentano, V.A., 1986, "The Pencil $(s E-A)$ and Controllability-Observability for Generalized Linear Systems: a Geometric Approach," SIAM J. Control and Optimization, Vol. 24, No. 4, pp. 616-638.

3. Astrom, K.J., 1983, "Theory and Applications of Adaptive Control-A Survey," Automatica, 19, pp. 471-481.

4. Bender, J., and Laub, A.J., 1987, “The LinearQuadratic Optimal Regulator for Descriptor System," IEEE Trans. on Automatic Control, Vol. AC-23, No. 1, pp. 672-688.

5. Cobb, D.J., 1983, "Descriptor Variable Systems and Optimal State Regulation," IEEE Trans. Automatic Control, Vol. AC-28, No. 5, pp. 601611.

6. Cobb, D.J., 1984, “Controllability, Observability, and Duality in Singular Systems," IEEE Trans. on Automatic Control, Vol. AC-29, No. 12, pp. 1076-1082.

7. Doyle, J.C., Glover, K, Khargonekar, P.P., and Francis, B.A., 1989, "State-Space Solutions to Standard $\mathrm{H}_{2}$, and $\mathrm{H}_{\infty}$ Control Problems," IEEE Trans. on Automatic Control, Vol. AC-34, No. 8, pp. 831-846.
8. Joshi, S.M., 1989, Control of Large Flexible Space Structures (Lecture Notes in Control and Information Sciences Vol. 131), New York: Springer-Verlag.

9. Kawamoto, A., and Katayama, T., 1996, “The Dissipation Inequality and Generalized Algebraic Riccati Equation for Linear Quadratic Control Problem of Descriptor System," 1996 IFAC 13th World Congress, San Francisco, USA, pp. 103108.

10. Knobloch, H.W., Isidori, A., and Flockerzi, D., 1993, Topics in Control Theory. Birkhäuser, Basel, Switzerland.

11. Lewis, F.L., 1986, "A Survey of Linear Singular Systems," Circuits, Systems, Signal Process, Vol. 5, No. 1, pp. 3-36.

12. Narendra, K.S., and Taylor, J.H., 1973, Frequency Domain Criteria for Absolute Stability, Academic, New York.

13. Popov, V.M., 1973, Hyperstability of Control Systems, Berlin: Springer-Verlag.

14. Safonov, M.G., Jonckheere, E.A., Verma, M., and Limebeer, D.J.M., 1987, "Synthesis of Positive Real Multivariable Feedback Systems," Int. J. Control, Vol. 45, No. 3, pp. 817-842.

15. Sun, W., Khargonekar, P.P., and Shim, D., 1994, "Solution to the Positive Real Control Problem for Linear Time-Invariant Systems," IEEE Trans. on Automatic Control, Vol. AC-39, No. 10, pp. 2034-2046.

16. Takaba, K., Morihira, M., and Katayama, T., 1994, " $H_{\infty}$ Control for Descriptor Systems -A JSpectral Factorization Approach," Proceeding of $33 r d$ Conference on Decision and Control, Lake Buena Vista, FL, December, pp. 2251-2256.

17. Wang, H.S., Yung, C.F., and Chang, F.R., 1997, "A State Space Approach to Youla Parameterization for Descriptor Systems," submitted for publication.

18. Wang, H.S., Yung, C.F., and Chang, F.R., 1998, "Bounded Real Lemma and $H_{\infty}$ Control for Descriptor Systems," IEE Proceeding D: Control Theory and Its Applications, Vol. 145, No. 3, pp. 316-322.

19. Wang, Y.Y., Frank, P.M., and Clements, D.J., 1993, "The Robustness Properties of the Linear Quadratic Regulators for Singular Systems," IEEE Trans. Automatic Control, Vol. AC-38, No. 1, pp. 96-100.

20. Willems, J.C., 1971, "Least Square Stationary Optimal Control and the Algebraic Riccati Equation, "IEEE Trans. on Automatic Control, Vol. AC16, No. 6, pp. 621-634.

21. Yung, C.F., 1999, "State-Space Characterization of All Solutions to a Positive Real Control Problem," J. Chinese Institute of Engineers, Vol. 
22, No. 5, pp. 653-659.

22. Zames, G., 1966a, "On the Input-Output Stability of Time-Varying Nonlinear Feedback Systems-Part 1: Conditions Derived Using Concepts of Loop Gain, Conicity and Positivity," IEEE Trans. Automatic Control, Vol. AC-11, No. 2, pp. 228-238.

23. Zames, G. 1966b, "On the Input-Output Stability of Time-Varying Nonlinear Feedback SystemsPart 2: Conditions Involving Circles in the Frequency Plane and Sector Nonlinearities," IEEE
Trans. Automatic Control, Vol. AC-11, No. 3, pp. 465-476.

Discussions of this paper may appear in the discussion section of a future issue. All discussions should be submitted to the Editor-in-Chief.

Manuscript Received: Jun. 04, 1999

Revision Received: May 31, 2000

and Accepted: Jul. 26, 2000

\title{
描述系統之正實控制問題與廣義代數 Riccati \\ 方程式
}

\author{
王和盛 \\ 工業技術研究院航空與太空發展中心 \\ 容志輝 \\ 國立台灣海洋大學電機工程學系 \\ 張帆人 \\ 國立台灣大學電機工程學系
}

\begin{abstract}
摘 要
本文首先探討描述系統的廣義代數 Riccati 方程式以及廣義正實引理的一 些性質。以這些結果爲基礎, 本文的主要目的是求解描述系統正實控制問題的 所有穞定控制器。描述系統輸出正實控制問題有解的充分必要條件爲兩個廣義 代數 Riccati 方程式有穩定解。這一結果可視爲由 Sun 等人（1994）所得代數 Riccati方程式結果的推廣。在正實控制問題有解的情況下, 本文給出所有解的 狀態空間表示式。

關鍵詞：正實控制，描述系統，廣義代數 Riccati 方程式。
\end{abstract}

